\title{
Optimal Earth's reentry disposal of the Galileo constellation
}

\author{
Roberto Armellina,1,*, Juan F. San-Juan ${ }^{\mathrm{b}, 2}$ \\ ${ }^{a}$ Surrey Space Centre, University of Surrey, BA Building, Guildford GU2 7XH, United \\ Kingdom \\ ${ }^{b}$ GRUCACI, University of La Rioja, 26004 Logroño, Spain
}

\begin{abstract}
Nowadays there is international consensus that space activities must be managed to minimize debris generation and risk. The paper presents a method for the end-of-life (EoL) disposal of spacecraft in Medium Earth Orbit (MEO). The problem is formulated as a multiobjective optimization one, which is solved with an evolutionary algorithm. An impulsive manoeuvre is optimised to reenter the spacecraft in Earth's atmosphere within 100 years. Pareto optimal solutions are obtained using the manoeuvre $\Delta v$ and the time-to-reentry as objective functions to be minimised. To explore at the best the search space a semi-analytical orbit propagator, which can propagate an orbit for 100 years in few seconds, is adopted. An in-depth analysis of the results is carried out to understand the conditions leading to a fast reentry with minimum propellant. For this aim a new way of representing the disposal solutions is introduced. With a single $2 \mathrm{D}$ plot we are able to fully describe the time evolution of all the relevant orbital parameters as well as identify the conditions that enables the eccentricity build-up. The EoL disposal of the Galileo constellation is used as test case.
\end{abstract}

Keywords: Trajectory Optimization; End-of-life disposal; Navigation Constellation; Space debris; Long-term propagation;

\footnotetext{
*Corresponding author (tel: +44 (0)1483 68 6024)

Email addresses: r.armellin@surrey.ac.uk (Roberto Armellin), juanfelix.sanjuan@unirioja.es (Juan F. San-Juan)

${ }^{1}$ Senior Lecturer

${ }^{2}$ Associate Professor
} 


\section{Introduction}

Over the recent years, the risk posed to space based activities has increased to a worrying level due to the number of uncontrolled manmade objects (Space Debris) in Earth orbit. This Earth-orbiting space debris consists of spent upper and launch stages, fragments and inactive satellites. There are currently measures in place to minimise the amount of debris left in orbit and risk of further fragment-producing collisions that would have a devastating impact on the space environment. As well as disposal and debris mitigation strategies being implemented into the design process of satellites, organisations such as the Inter-Agency Space Debris Coordination Committee and the UN COPUOS provide debris mitigation guidelines to ensure that our presence and activities in space are continued safely.

The Medium Earth Orbit (MEO) region of near-Earth space is a large region of space that ranges from $2000 \mathrm{~km}$ (the upper boundary of Low Earth Orbit, LEO) to $35786 \mathrm{~km}$ (the lower boundary of Geostationary Earth Orbit, GEO). It is best known today for Global Navigation Satellite Systems (GNSS) use, whilst historically a significant number of its residents have been in highly elliptical orbits that spend a majority of each revolution in this region (e.g., Molniya). The MEO region is home not only to satellites, but fragments and spent upper stages of many launch vehicles. Due to the low spatial densities and low collision probabilities that are currently associated with it, the MEO region is not yet a protected region of space. However, two main factors have stimulated the study of the long-term dynamics of MEO objects from a space debris perspective: (1) this region is becoming of even more strategic importance (and thus more crowded) due to the launch of Galileo and Beidou constellations; (2) the occurrence of resonances due to Earth's geopotential and luni-solar perturbations may cause instability in the recommended graveyard orbits (Chao, 2000) resulting in the urge to re-think the end-of-life (EoL) disposal of these spacecraft.

As a result, there is a huge body of literature on MEO dynamics by researchers belonging to both the astrodynamics and celestial mechanics communities. Three main objectives have driven the research from the astrodynamics standpoint:

1. Studying stability of operational orbits to estimate the station keeping requirements (Chao and Schmitt, 1990; Deleflie et al., 2005; NavarroReyes et al., 2009); 
2. Understanding the stability of disposal orbits for GNSS with an eye on the long-term risk posed to operational satellites (Chao, 2000; Jenkin and Gick, 2002; Chao and Gick, 2004; Saunders et al., 2005; Rossi, 2008; Deleflie et al., 2011; Pardini and Anselmo, 2012; Radtke et al., 2015);

3. Exploiting the long-term effect of the perturbations to design low-cost Earth's reentry EoL disposals (Jenkin and Gick, 2005; Merguizo Sanchez et al., 2010; Alessi et al., 2014; Merguizo Sanchez et al., 2015; Alessi et al., 2016; Rosengren et al., 2017);

(Note that sometimes in a same work both points 2) and 3) were studied, thus the proposed classification should be taken with some flexibility.) Works with a more celestial mechanics focus studied the dynamics from a more theoretical point of view, with the aim of explaining the intricate dynamical behaviour (Hughes, 1980, 1981; Rosengren et al., 2015; Stefanelli and Metris, 2015; Lara et al., 2014; Celletti et al., 2016; Celletti and Galeç, 2016), particularly highlighting the overlapping of different resonances (Daquin et al., 2016; Rosengren et al., 2015) leading to chaotic motion and therefore the need to treat the disposal trajectories in a statistical manner (Rosengren et al., 2017).

Despite the large amount of literature, only a few works looked at the problem from the spacecraft (i.e., disposal manoeuvre) point of view. Most of them in fact analysed the eccentricity build-up by running simulations on a grid of initial conditions and reference epochs, without translating the change of initial conditions into spacecraft manoeuvres (exceptions are found in Merguizo Sanchez et al. (2015); Radtke et al. (2015); Alessi et al. (2014)). Fixed and uniformly discretised grids help in building a global picture of the dynamical behaviour as a function of different parameters, but bring the disadvantage that all the regions of the phase space are analysed with the same depth, while a mission designer would prefer to gain more details on the most interesting ones, e.g., those leading to reentry. Moreover, in order to limit the computational burden, some parameters have been kept fixed and the remaining one either sampled on relatively coarse grids or on reduced search spaces (e.g., in Alessi et al. (2016) a 3D grid with 10 deg separation on angular variables and approximately one year in time is used).

It is well understood that the eccentricity build-up is caused by inclinationdependent resonances due to Earth's geopotential and luni-solar perturbations (a exhaustive review of this phenomenon is given in Rossi (2008), and 
an elegant analysis is carried out in Daquin et al. (2016)). On the other hand, due to the complexity of the dynamics, researchers have often focused their attention only on a particular aspect of the problem or used several simplifications to deal with the problem analytically: e.g., neglecting either the Moon or Sun perturbations; analysing only isolated resonant terms in the Hamiltonian of the problem; and considering only $J_{2}$ to describe the evolution of the argument anomaly of perigee $\omega$ and the longitude of the node $\Omega$. Many works (probably to date Alessi et al. (2016) contains the most exhaustive numerical analysis on these aspects) have highlighted the key role played by the initial value of $\omega, \Omega$, and inclination $i$, as well as the initial configuration of the Moon. However, there is a lack of explanation on why it is so, and more importantly the coupling among these parameters are not discussed in details. Finally, while it is agreed that a higher initial eccentricity promotes the eccentricity build-up, is not clear whether the initial semi-major axis $a$ should be lowered (e.g., Alessi et al. (2016) and all following papers published by that research team using the same simulations) or increased (e.g., Merguizo Sanchez et al. (2015)). All these considerations represent motivations for this work, whose main contributions are listed hereafter.

The search for eccentricity build-up solutions is formulated as an optimization problem, with the goal of minimising the disposal manoeuvre (building on a previous study on INTEGRAL spacecraft disposal by Armellin et al. (2015)). Using a population-based stochastic optimiser (Kennedy and Eberhart, 2001) to scrutinise the search space brings the advantage of performing an in-depth analysis only of those regions leading to a reentry, whereas the least interesting ones are quickly pruned away. By defining the search space on the manoeuvre $\Delta v$ the solutions are directly endowed with feasibility properties. In this respect, in this work it is chosen to extend the search space to high values of $\Delta v$ with the goal of computing fast reentry options that could be targeted, in the future, by low-thrust propulsion (a preliminary analysis on this aspect is presented in Alessi et al. (2014)). Moreover, requirements on reentry time are directly taken into account by formulating the EoL disposal as a multiobjective optimisation problem, in which the reentry time is an additional objective to be minimised together with the disposal $\Delta v$. Lastly, the optimal $\Delta v$ can be transformed into optimal initial variation of orbital parameters, enabling an analysis of the phase space with a very fine discretisation. The effect of the Moon's configuration on the disposal $\Delta v$ is studied by running additional optimisations, in which the disposal epoch is left free to change for 18.6 years (i.e., for en entire revolution of the Moon's 
node).

The need of carrying out large scale optimisations in reasonable amount of time has stimulated the search for fast and accurate propagators. Different tests were carried out with the outcome of selecting a single-averaged and a double-averaged dynamical model (including only Earth's geopotential and luni-solar perturbations), which enables the propagation for 100 years in less than 10 and 1 seconds, respectively. In this context our work is similar to those by Daquin et al. (2016) and Lara et al. (2014), with the additional value that the solutions are validated with a high fidelity numerical propagator.

The large variety of disposal solutions obtained with the optimisation approach prompted us to work on an in-depth understanding of the dynamics (however, limited to relatively fast and low $\Delta v$ eccentricity build-up scenarios). It is found that the expressions for the eccentricity derivative provided by in Chao and Gick (2004) fully capture the physics of the problem. In particular, starting from these expressions we will show that 1) an initial increase of semi-major axis is always preferable for low-cost solutions and 2) the eccentricity build-up is always associated with the occurrence of an inclination-dependent resonance due to the non-spherical shape of the Earth and luni-solar attraction (in full agreement with the literature), but also non-resonant terms (e.g., the $2 \omega$ term at Galileo inclination) do play a key role in the eccentricity growth. Based on these analyses we are able to identify the initial values of $\Omega$ that are more favourable for eccentricity growth and explain the relations between both the initial $\Omega$ and $\omega$, and the initial $i$ and $\Omega$ that maximise the eccentricity build-up. Moreover, it is clarified how the Moon's configuration (and in particular the Moon's inclination) can contribute in limiting or enhancing the eccentricity increase. Lastly, a new way of representing the disposal trajectories is presented, a single figure with which all the relevant elements of a disposal can be explained.

The remainder of the paper is organised in three main parts: the formulation of the problem given in Sec 2, 3, and 4; optimisation results and validations summarised in Sec. 5, 6, and 7; explanation of the results in Sec. 8 and Sec. 9. In the first part we start by introducing the different dynamical models used in this work: a single- and double-averaged semi-analytical (SA) propagators based on Lie transforms and a high accuracy numerical propagator. The first part is completed by a description of the optimisation problem formulation and the definition of the test case: the EoL reentry disposal of the Galileo constellation. In the second part, the results of the optimisations for fixed Moon's configuration are presented first. This is followed by an 
analysis of the sensitivity of the optimal trajectories to Moon's configuration and a set of optimisations with free Moon's configuration. The second part is completed by the validation of the results in a high fidelity dynamical model. In the third part we explain what are the key elements that determine the eccentricity build-up, in particular the favourable initial values of the orbital parameters, and the relation between them and the Moon's configuration. The third part includes the presentation of the disposal map, an attempt to explain such an intricate problem with just a two-dimensional plot. Final remarks are made in Sec. 10.

\section{Trajectory Propagation}

Three different propagators are used in this study, two SA and one numerical. To reduce the computational cost of the EoL disposal optimisations both a single-averaged and a double-averaged semi-analytical propagators are used. The Accurate Integrator for Debris Analysis (AIDA) is the numerical propagator used to assess the accuracy of SA propagators and the impact of the neglected perturbations (e.g atmospheric drag and solar radiation pressure).

\subsection{Numerical propagator}

The perturbations included in AIDA (Morselli et al., 2014) are the geopotential acceleration, atmospheric drag, solar radiation pressure, and thirdbody perturbations. The gravitational model selected for the numerical propagator is EGM2008 (Pavlis et al., 2012). The model combines gravitational information from GRACE with surface data and is complete to spherical harmonic degree and order 2160. The field model was downloaded from the International Centre for Global Earth Models (ICGEM) website ${ }^{3}$. The default degree $n$ and order $m$ for the gravitational harmonics are set to 10 based on a trade-off between accuracy (higher fidelity with respect to the SA propagators) and computational time.

The computation of the perturbing acceleration due to atmospheric drag is based on the Naval Research Laboratory's Mass Spectrometer and Incoherent Scatter Radar of year 2000 (NRLMSISE-00) model (Picone et al., 2002). This model includes the anomalous oxygen component together with Helium,

\footnotetext{
${ }^{3}$ http://icgem.gfz-potsdam.de/ICGEM/
} 
atomic and molecular Oxygen, atomic and molecular Nitrogen, Argon, and Hydrogen. The model requires as inputs the solar and geomagnetic activity, geodetic altitude and latitude, longitude, year, day, and time of day in UT. Solar and geomagnetic data are read from up-to-date space weather files that are automatically downloaded from CelesTrack ${ }^{4}$.

The gravitational attraction of the Sun and the Moon is based on NASA JPL's DE430 ephemeris (Standish, 1998). The same ephemeris model is used to account for the contribution due to solar radiation pressure. The spherical assumption is made for the spacecraft, thus the the resulting acceleration is in the direction of the Sun-satellite vector. Dual-cone shadow model is adopted.

\subsection{Single- and double-averaged $S A$ propagators}

The SA theory is constructed using Deprit's algorithm by Lie transforms (Deprit, 1969), in which short-periodic terms related to the mean anomaly $M$ of the satellite are removed by an averaging process. In the case of third body perturbation, these transforms are carried out in closed form of the eccentricity by introducing the eccentric anomaly of the satellite $E$ and using the relations $r \sin \nu=a \sqrt{1-e^{2}} \sin E$ and $r \cos \nu=a(\cos E-e)$, in which $r$ is the orbital radius, $a$ is the semi-major axis, $e$ is the eccentricity and $\nu$ the true anomaly. To carry out the averaging, the differential relation $\mathrm{d} M=(r / a) \mathrm{d} E$ is used, which is obtained from the Kepler equation noting that $r=a(1-e \cos E)$. This allows the theory to remain valid for all eccentricities lower than one.

The perturbations included in this model are the Earth $J_{2-8}$ zonal harmonics and lunisolar perturbations. The modelled lunisolar perturbation relies on Chapront's analytical ephemerides (Chapront-Touze and Chapront (1988) and Chapront and Francou (2003)), which have a sufficient precision for the purpose of this study.

The disturbing effects of the third-body perturbations are modelled by the first term of the Legendre polynomial expansion for the Sun, whilst it is also expanded to the fifth term when considering perturbations from the Moon. The resulting Hamiltonian has two degree of freedom and it is timedependent, due to the third-body perturbations.

This single-averaged propagator allows the easy propagation of long-term orbits in the order of seconds as opposed to several minutes (as for AIDA)

\footnotetext{
${ }^{4}$ http://celestrak.com/SpaceData/SpaceWx-format.asp
} 
in the case of a numerical propagation method.

In a second step, the efficiency of the single-averaged semi-analytical propagator is improved by removing the mean anomaly of the Moon. This simplification is possible because in the MEO region there are neither apsidal nor nodal resonances with the Moon's mean motion (Breiter, 2001). The closed form is also possible for the averaging over the mean anomaly of the Moon, now resorting to the differential relation $r_{M}^{2} \mathrm{~d} \nu_{M}=a_{M}^{2} \sqrt{1-e_{M}^{2}} \mathrm{~d} M_{M}$, and expressing the radius of the Moon as $r_{M}=\frac{a_{M}\left(1-e_{M}^{2}\right)}{1+e_{M} \cos \nu_{M}}$. After this transform, the system still has two degrees of freedom, but now it is time independent. As a result the propagations required in this work can be carried out in less than a second.

The interested reader may refer to Lara et al. (2014, 2012) for more details.

\section{Optimisation of EoL reentry disposal}

The goal of the optimisation of reentry disposal is to find a set of Pareto optimal solutions with respect to two objective functions minimised: the disposal $\Delta v$ and the time-to-reentry. The minimisation of the $\Delta v$ is driven by the need to design disposal manoeuvres with minimum propellant consumption; whereas short-time reentries are attractive from both collision risk and operations standpoints.

The definition of the optimisation problem is rather simple. The spacecraft's initial conditions are taken from an available two-line element. These initial conditions are converted into state vectors $\left(\boldsymbol{r}_{0}, \boldsymbol{v}_{0}\right)$ using SGP4 theory (Hoots and Roehrich, 1980), and propagated forward in time to a time $t$ with the selected propagator. At time $t$ the disposal manoeuvre is applied. This manoeuvre is defined by the $\Delta v$, the maneuver azimuth and elevation angles, $\alpha$ and $\delta$, and the true anomaly at execution, $\nu$ (considered here independent from $t$ as we work with averaged quantities). It is to be noted that $\alpha=\tan ^{-1}\left(\Delta v_{y} / \Delta v_{x}\right)$ and $\delta=\sin ^{-1}\left(\Delta v_{z} / \Delta v\right)$ are defined in a local reference frame within which the $x$ and $z$ axes are aligned with the velocity and angular momentum vectors respectively. With this formulation the optimisation vector associated to a manoeuvre takes the form $\boldsymbol{x}=(\Delta v, \alpha, \delta, \nu, t)$. The bounds for the optimisation parameters are $\Delta v \in\left[0, \Delta v_{\max }\right], \alpha \in[0,2 \pi], \delta$ $\in[-\pi / 2, \pi / 2], \nu \in[0,2 \pi]$ and $t \in\left[t_{0}, t_{\max }\right]$.

The spacecraft's state is then propagated forward 100 years after the application of the disposal. In order for the spacecraft orbit to decay to 
Table 1: Reentry disposal objective function definition.

\begin{tabular}{ll} 
Condition & Objective Function \\
\hline $\min h_{p}>h_{\text {atm }}$ & $f_{1}(\boldsymbol{x})=\min h_{p}$ \\
$\min h_{p} \leq h_{\text {atm }}$ & $f_{2}(\boldsymbol{x})=\left(\Delta v, \Delta t_{R e}\right)$ \\
\hline
\end{tabular}

an altitude low enough for atmospheric reentry, the manoeuvre must set in motion a long-term increase in the eccentricity of the orbit. Doing so will cause the perigee altitude, $h_{p}$, to decay to a height low enough within the Earth's atmosphere for drag effects to dominate and initiate reentry. The long-term evolution of $h_{p}$ is therefore to be monitored until the target altitude is achieved. The altitude at which drag effects become dominant can be denoted by $h_{a t m}$. In this study, $h_{a t m}$ takes the value of $120 \mathrm{~km}$.

To force the optimiser into searching first for the lowest possible perigee altitude, a sequence of two different objective functions are defined. The first objective function is the lowest perigee altitude reached in 100 years if its value exceeds $h_{a t m}$. If the lowest spacecraft perigee altitude is below the atmospheric altitude, then a two dimensional objective function is optimised. The first component of the objective function is the $\Delta v$ required to perform the manoeuvre, the second one is the time-to-reentry $\Delta t_{R e}$. In this way we are able to build a Pareto optimal set of solutions, including high $\Delta v$ and fast reentry. The objective function formulation is summarised in Table 1 and shows the constraints used to drive the optimiser into finding Pareto optimal solutions.

The solver used in this work is a multiobjective particle swarm optimiser (Coello Coello and Lechuga, 2002) already adopted for the design of the disposal of INTEGRAL mission (Armellin et al., 2015).

The search space investigated for the $t$ variable is limited to two months, as the disposal manoeuvre should be applied reasonably close to the mission end, for both cost and failure risk minimisation. On the other hand, a maximum $\Delta v$ up to $600 \mathrm{~m} / \mathrm{s}$ is considered. We have decided to allow for unrealistically high values of $\Delta v$ for two main reasons: (1) to investigate a large set of initial orbital parameters; (2) the second generation of Galileo will likely use electric thrusters ${ }^{5}$, which will make high $\Delta v$ more affordable

\footnotetext{
${ }^{5}$ www.esa.int/Our_Activities/Navigation/Electric_thrusters_may_steer_ Galileo_in_future
} 
from the propellant consumption point of view.

As a final remark, a single disposal manoeuvre is considered in this work to limit the number of optimisation variables. Although this simple strategy is sufficient to trigger the eccentricity build-up, it is not excluded that the use of multiple manoeuvres, in particular separated by long ballistic arcs, could further reduce the overall disposal $\Delta v$.

\section{Test case: Galileo constellation}

Galileo is Europe's own global satellite navigation system with a proposed 30 satellites in MEO at an altitude of $23,222 \mathrm{~km}$. When complete, the system will be inter-operable with both the GPS and GLONASS satellite navigation systems whilst also delivering accuracies down to the meter. Ten satellites will occupy one of three orbital planes that are inclined at $56 \mathrm{deg}$ to the equator. These satellites are to be spread evenly around each of the three planes and have an orbital period of around 14 hours. Each plane contains two spare satellites, that can be brought into service should any other satellite fail.

In April 2016 (when the first version of this manuscript was written) 12 Galileo spacecraft were in orbit, 8 of which were fully operational positioning, navigation and timing satellites ${ }^{6}$. The first couple of spacecraft was launched in 2011, and the last couple was launched in December 2015. Due to a launcher failure, the first two fully operational Galileos (NORAD SSC code 40128 and 40129) were not inserted in the proper orbit, and are currently at lower inclination $(\approx 50 \mathrm{deg})$ and in highly eccentric orbit (apogee height of $26,048 \mathrm{~km}$ and perigee height of $17,152 \mathrm{~km}$ ). The ten spacecraft that reached the operational orbits are already spread on the three different planes. At the 28 of April 2016 four (SSC 37846, 37847, 40544 and 40545) have a $\Omega \approx 84 \mathrm{deg}$, four (SSC 38857, 38858, 41174 and 41175) have $\Omega \approx 203 \mathrm{deg}$, and two (SSC 40889 and 40890$) \Omega \approx 323 \mathrm{deg}$. As spacecraft with same $\Omega$ will produce similar results (the reader must consider the initial $\omega$ as a free parameter as the $\Delta v$ required to change its initial value is negligible for initial low eccentric orbits), our analysis is restricted to only three Galileo spacecraft placed on three different planes. Their osculating classical orbital parameters $(a, e, i, \Omega, \omega, M)$, obtained via SGP4 evaluation of two-line elements (TLE) ${ }^{7}$,

\footnotetext{
${ }^{6}$ www.gsc-europa.eu/galileo-gsc-overview/system

${ }^{7}$ retrieved from www.space-track.org
} 
Table 2: Test cases: osculating orbital parameters obtained from SGP4 evaluations of TLE at TLE epochs.

\begin{tabular}{lrrrr}
\hline \multicolumn{2}{c}{ SSC } & 37846 & 41175 & 40890 \\
\hline Epoch & JED & 2457506.996 & 2457494.638 & 2457492.942 \\
$a$ & $\mathrm{~km}$ & 29601.793 & 29598.896 & 29601.769 \\
$e$ & - & 0.000499 & 0.000173 & 0.000432 \\
$i$ & $\mathrm{deg}$ & 55.516 & 54.982 & 57.280 \\
$\Omega$ & $\operatorname{deg}$ & 83.329 & 203.549 & 323.785 \\
$\omega$ & $\mathrm{deg}$ & 0.541 & 272.857 & 40.226 \\
$M$ & $\operatorname{deg}$ & -0.535 & 166.264 & -40.193 \\
\hline
\end{tabular}

together with reference dates are listed in Table 2 .

\section{Optimal solutions}

Figure 1 shows the three Pareto fronts computed in 100 iterations with a swarm of 120 particles with the single-averaged propagator. 137 Pareto optimal solutions are computed for SSC 37846, 200 (the maximum number allowed in the Pareto set) for SSC 41175, and 149 for SSC 40890. In the remainder of the paper, in each Pareto front, the first solution is the solution with minimum $\Delta v$, whereas the last one is the one with fastest reentry.

Although the disposal manoeuvres are applied for the three objects roughly at the same time (same configuration of the Moon), the Pareto optimal solutions are significantly different. The minimum $\Delta v$ that allows for a reentry in less than 100 year ranges from $86.7 \mathrm{~m} / \mathrm{s}$ for SSC 40890 to $173.3 \mathrm{~m} / \mathrm{s}$ for SSC 37846. Considering a dry mass of around $670 \mathrm{~kg}$ and current hydrazine thrusters (specific impulse of $200 \mathrm{~s}$ ), the EoL manoeuvre will require a propellant mass in the range $[30,60] \mathrm{kg}$. For the next generation of Galileo this amount could be reduced by roughly one order of magnitude, even in the case of an increased spacecraft dry mass, if electric thrusters with specific impulse of 2,000 to 4,000 s will be selected. The minimum reentry time for the maximum $\Delta v$ of $600 \mathrm{~m} / \mathrm{s}$ ranges from 11.27 years for SSC 41175 to 64.04 years for SSC 37846. The maximum $\Delta v$ solutions require an unrealistic value of $230 \mathrm{~kg}$ of propellant with current thrusters. This value could be significantly 


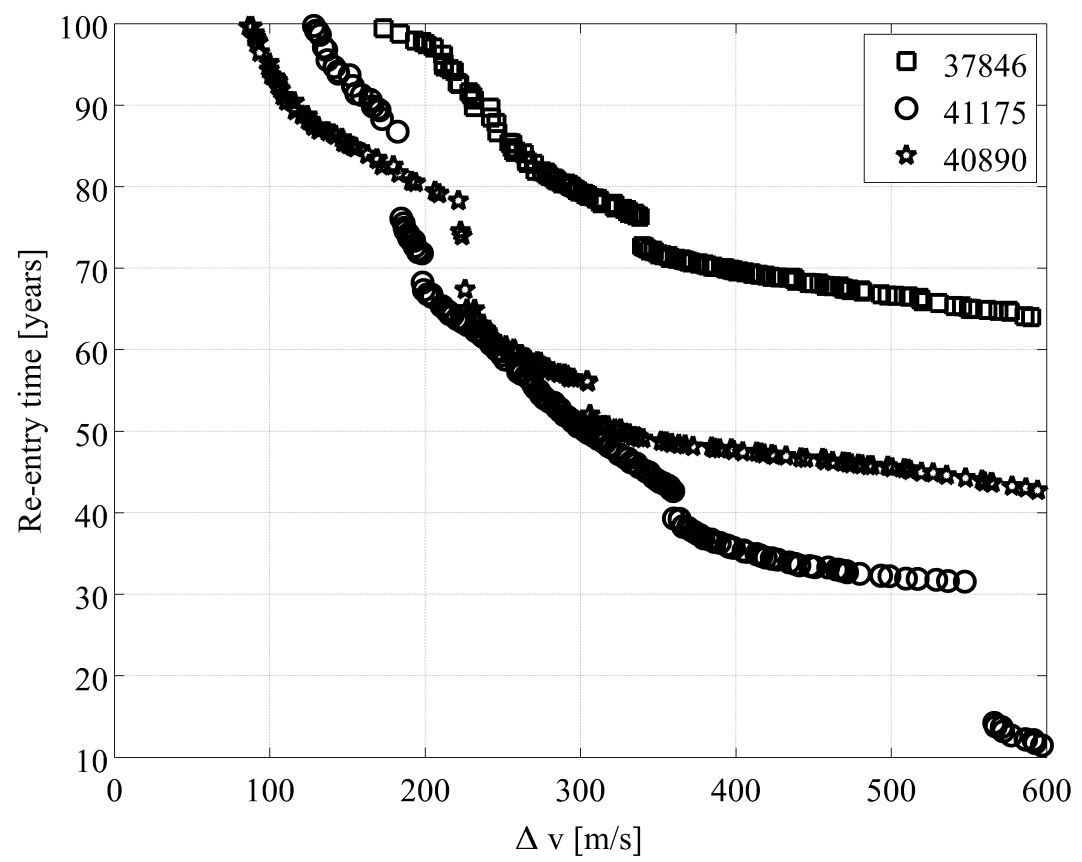

Figure 1: Atmospheric reentry Pareto front obtained with single-averaged SA propagator.

lower in case of electric thrusters (even considering an increased dry mass), making a relatively fast reentry a viable option for the future generation of Galileo. It is worth noting that for all the spacecraft the same disposal windows were considered (i.e., same configuration of third bodies) and that the spacecraft share similar orbital parameters with only exception of $\Omega$ : this clearly highlights the importance of the initial $\Omega$ for the disposal design.

In Fig. 2-4 all the Pareto optimal solutions for the three spacecraft are analysed in more depth. In particular, for each spacecraft we plot the perigee and apogee altitude $\left(h_{p}\right.$ and $\left.h_{a}\right)$ profiles, the trajectories in the $e^{-}(2 \omega+$ $\Omega)$ plane, and the changes in the semi-major axis, eccentricity, and $2 \omega+$ $\Omega$ imparted by the manoeuvres. In these plots the pre-disposal values are indicated by a up-pointing triangles and post-manoeuvre values by downpointing triangles. The grey scale is used to differentiate the different disposal $\Delta v$, with a darker line indicating a lower value. First of all it is worth noting that adjacent Pareto optimal solutions are characterised by variations of (all) orbital parameters that are much smaller than the minimum grid sizes 
considered in the literature (e.g., in Alessi et al. (2016)), further justifying the use of an optimiser.

There are several commonalities among the solutions:

1. The optimal manoeuvres increase both the initial eccentricity and semimajor axis of the orbit (note that a same increase in the eccentricity can be obtained by a reduction of the semi-major axis, but this option is never selected by the optimiser);

2. The minimum value of the $\Delta v$ is determined by the minimum required increase of initial eccentricity (minimum for SSC 40890 and maximum for SSC 37846);

3. The higher the initial eccentricity and semi-major axis are, the faster the reentry will be;

4. The optimal manoeuvres significantly change the initial value of $2 \omega+\Omega$;

5. The change of the initial $2 \omega+\Omega$ is achieved by varying the initial $\omega$, but leaving the $\Omega$ unchanged to minimise the $\Delta v$ consumption (this finding cannot be appreciated from the reported figures but by analysing the data);

6. A steep increase in the eccentricity during disposal is always associated to an almost constant value of the $2 \omega+\Omega$ in the range $[-110,-70]$ deg; i.e., at the occurrence of the so-called 2:1 resonance (Chao and Gick, 2004);

7. Slow reentries can be characterised by a permanence in the LEO regime (highlighted with a dashed red line in the perigee plots) for up to ten years, whereas for short reentry the permanence is lowered by one order of magnitude;

8. For all the solutions there is an extended phase in which the apogee is above the GEO region (highlighted with a dashed red line in the apogee plots), with potential fast intersections with the GEO protected region.

In addition to the differences in both the disposal $\Delta v$ and reentry times (highlighted by the Pareto fronts), two other dissimilarities can be highlighted from Fig. 2-4:

1. Fast reentry disposals exploit a significant variation of the inclination (in the direction of the critical inclination) only for SSC 37846 and 40890;

2. For SSC 37846 the eccentricity always decreases at the beginning of the disposal. 
It is worth remarking that Fig. 2-4 show that optimal solutions are characterised by fine variations of the initial orbit parameters, variations that would not be fully captured by more classical grid approaches.

\section{Effect of the Moon's configuration}

In the optimisations presented in Sec. 5 the disposal epoch was left free to change in a very short window to simulate a real scenario (i.e., at the EoL space operators cannot wait years before implementing a disposal manoeuvre). This has the drawback of not allowing us to highlight the effect of the Moon's configuration on disposal.

To quantify the effect of the Moon's configuration on the eccentricity build-up, the solutions with lowest $\Delta v$ for the three spacecraft (Sol \#1 of the three Pareto optimal sets) are analysed. Propagations are run for 100 years for fixed initial state, but varying the disposal date on a grid covering one full revolution of the Moon's node ( $\approx 18.6$ years). The results are shown in Fig. 5(a)-5(c) in which the grey scale is used to highlight the initial variation of the Moon's node with respect to its nominal value (a lighter curve indicates a larger change in the initial $\left.\Omega_{M}\right)$. As already noticed in several works (e.g. in Alessi et al. (2016); Lara et al. (2014); Rosengren et al. (2017)) the configuration of the Moon can cause large variations in the maximum eccentricity, up to 0.4 for SSC 40890. In addition, it can be highlighted that solutions with lower $\Delta v$ (i.e. lower initial eccentricity) are much more sensitive, as for example noticed in Celletti and Galeç (2016). However, it must be stressed that in these simulations the optimal solutions were computed for the nominal node of the Moon; thus, for each Moon's configuration new optimal reentry solutions should be recomputed to draw conclusions on the existence of low $\Delta v$ reentry solutions at different epochs.

A second analysis is then carried out in which Pareto optimal solutions are computed by letting the disposal epoch vary up to 18.6 years (but not changing the orbital elements of the spacecraft in this window); i.e., the algorithm can optimise the Moon's configuration. The obtained Pareto optimal solutions are illustrated in Fig. 5(d). A comparison with Fig. 1 reveals that the configuration of the Moon does play a role in eccentricity build-up. However, the spacecraft ranking in terms of minimum $\Delta v$ remains unchanged, with highest $\Delta v$ for SSC 37846 and lowest for SSC 40890. Moreover, the values of the minimum $\Delta v$ remains basically unchanged for these two spacecraft; whereas the one for SSC 41175 reduces from 128.4 to $102.6 \mathrm{~m} / \mathrm{s}$. 


\section{Validation of the results}

The results of the multiobjective optimization were obtained with the single-averaged dynamical model. This section has two objectives: (1) understand whether a simpler dynamical model can be used (the importance of this question has already been underlined by e.g. Daquin et al. (2016) and Gkolias et al. (2016)); (2) assess the accuracy of the solutions found with SA propagators. The first point is addressed first. The double-averaged dynamical model presented in Sec. 2.2 propagates initial conditions for 100 years in less than a second, thus the multiobjective optimisation can be run in the same amount of time with a much higher number of particles and iterations with respect to the single-averaged dynamical model. Figure 6 shows the Pareto front obtained with the double-averaged dynamical model using 1000 particles and 60 iterations. By comparing Fig. 6 with Fig. 1 it is clear that similar results are obtained with the two different propagators.

To further prove that the double-averaged dynamics are sufficiently accurate to design the disposal manoeuvre, the differences in the eccentricity between the two models are plotted for all the Pareto solutions of SSC 37846 in Fig. 7(a), in which $\bar{e}$ and $\overline{\bar{e}}$ indicate respectively the single- and doubleaveraged eccentricities. Noticeably the maximum relative difference is always smaller than 0.01, despite the single-averaged initial state has been used as initial condition for the double-averaged dynamical model.

To assess the accuracy of the solutions obtained with SA propagators, all the Pareto optimal trajectories are propagated with AIDA in a $10 \times 10$ Geopotential model, Sun and Moon third bodies perturbation, solar radiation pressure and atmospheric drag (area-to-mass ratio $A / m=1 \times 10^{-2} \mathrm{~m}^{2} / \mathrm{kg}$, drag coefficient $C_{D}=2.2$, and reflectivity coefficient $C_{R}=1.31$ ). The simulations were halted at perigee altitude of $120 \mathrm{~km}$. Figure $7(\mathrm{~b})$, in which $e$ indicates the eccentricity obtained with AIDA, shows that the maximum relative difference between AIDA and the single-averaged dynamical model is always below 0.05 (with a maximum close to the reentry phase when in AIDA the effect of drag becomes relevant), despite the single-averaged initial state has been used as initial condition for AIDA. In addition, according to AIDA, all the disposals did indeed reenter in the atmosphere, proving that the disposal manoeuvre can be designed with an averaged dynamical model, neglecting both the atmospheric drag and the solar radiation pressure.

As a final note it is worth mentioning that recent works have underlined the chaotic behaviour of the eccentricity build-up disposal trajectories (e.g., 
(Daquin et al., 2016; Celletti et al., 2016; Rosengren et al., 2017)) suggesting that approaches based on ensembles of trajectories would be more appropriate than individual-based ones. However, it must be recognised that for practical applications the sensitivity to initial conditions should be performed by selecting the amount of uncertainty in the initial conditions compatible with those of the problem at hand; e.g., given by the accuracy of the orbit determination process and the implementation of the disposal manoeuvre. Although this topic is out of the scope of this paper, the results obtained by validation with AIDA, in which the initial mean orbital elements are considered as osculating (equivalent to introducing a perturbation in the initial conditions) and perturbations neglected in the SA propagators are included, allows us to be confident in the actual flyability of the trajectories without the need of extended, thus unpractical, trajectory control.

\section{Analysis of eccentricity build-up}

This section is fully devoted to the explanation of the effect of the spacecraft initial conditions and Moon's configuration on the design of optimal EoL disposal trajectories. By performing numerical tests on the optimal disposal trajectories, it is found that the simple model presented by Chao and Gick (2004) is sufficient to represent the average evolution of $\dot{e}$ during the disposal. (In these tests we compared the $\dot{e}$ computed by the SA propagator with the one obtained by evaluating Eq. 1 on the calculated trajectories.) This model is further simplified here by neglecting the first trigonometric term (the one depending on $2(\omega-\Delta \Omega$ ), which is found to be negligible in all the range of inclination of interest), leading to the expression:

$$
\dot{e}=C_{1} \sin (2 \omega-\Delta \Omega)+C_{2} \sin 2 \omega+C_{3} \sin (2 \omega+\Delta \Omega)+C_{4} \sin 2(\omega+\Delta \Omega),
$$

in which $C_{i}=-\frac{15}{8} n_{3}^{2} R_{m} \frac{e \sqrt{1-e^{2}}}{n} K_{i}, n$ is the mean motion of the object, $n_{3}$ is the mean motion of the third body, $R_{m}$ is the mass ratio ( 1 for solar perturbation, $1 / 82.3$ for lunar perturbation), and $\Delta \Omega=\Omega-\Omega_{3}$ is the difference between the longitude of the node of the satellite and the third body. (Note that due to a typo in Chao and Gick (2004), in which $R_{m}=182.3$, all the following works have reported the wrong coefficient for the mass ratio of the Moon.) It is important to note that both the $i_{3}$ and $\Omega_{3}$ are referred to the equatorial plane, thus for the Sun it can be assumed $i_{S}=23.43$ and $\Omega_{S}=0$ 
deg, whereas for the Moon $i_{M} \in[18.14,28.72] \mathrm{deg}$, and $\Omega_{M} \in[-13.44,13.42]$ deg with periodic behaviour dictated by nodal regression period $(\approx 18.6$ years). As $\Omega_{S}=0$ and $\Omega_{M}$ oscillates around zero, in a first analysis we replace $\Delta \Omega$ with $\Omega$ to in Eq. (1).

The expressions for the four inclination-dependent coefficients $K_{i}$ are

$$
\begin{aligned}
K_{1} & =\frac{1}{2} \sin i \sin 2 i_{3}(\cos i-1), \\
K_{2} & =\sin ^{2} i\left(3 / 2 \sin ^{2} i_{3}-1\right), \\
K_{3} & =\frac{1}{2} \sin i \sin 2 i_{3}(1+\cos i), \\
K_{4} & =\frac{1}{2} \sin ^{2} i_{3}\left(1 / 2 \sin ^{2} i-\cos i-1\right) .
\end{aligned}
$$

The multiplicative coefficients $n_{3}^{2} R_{m}$ of the Sun and the Moon are of the same order of magnitude, with the one of the Moon approximately 2.2 bigger. As a result, both the contributions of the Sun and the Moon shall be accounted for when studying the eccentricity growth of GNSS spacecraft. The values of the coefficients $K_{i}$ are reported in Fig. 8 for a range of spacecraft inclination values and different values of $i_{M}$ (highlighted by the gray scale).

$K_{2}$ and $K_{3}$ are the dominating coefficients and they shall be accounted for when studying eccentricity build-up scenarios. $K_{2}$ is dominant at higher inclination, and can explain why, for SSC 37846 and 40890, fast reentries are achieved by a significant increase of the initial inclination. As highlighted by the gray bands, the effect of $i_{M}$ on the values of $K_{i}$ is significant. In particular, this graph explains that, at Galileo inclinations, the inclination of the Moon is determinant in establishing the dominant amplitude in Eq. (1), a key point in the long-term evolution of the eccentricity. This proves that the actual inclination of the Moon shall be used for quantitative studies, whereas an average value (the node regression period is short with respect to the disposal window) can be considered in qualitative studies.

The multiplicative term $e \sqrt{1-e^{2}} / n$ in the $C_{i}$ coefficients increases with both the semi-major axis and eccentricity. An increase in the initial semimajor axis (note that its mean value remains constant during the disposal) produces an increase of $1 / n$, as from Kepler's third law $\mu=a^{3} n^{2}$. The function $f(e)=e \sqrt{1-e^{2}}$ is monotonically increasing on $[0,1 / \sqrt{2}]$ to reach the maximum value $f(1 / \sqrt{2})=1 / 2$. This explains why disposal manoeuvres will tend to increase both the semi-major axis and eccentricity as already shown in Fig. 2(c), 2(d), 3(c), 3(d), 4(c), 4(d). It is worth mentionionig that a same variation of the initial eccentricity can be obtained with a reduction of the semi-major axis (with the advantage of moving the perigee closer to 
the reentry condition), but these solutions are then characterised by a lower eccentricity growth. This result is in agreement with Merguizo Sanchez et al. (2015), in which it is shown that, when designing reentry disposals, an increment of the semi-major axis by $10,000 \mathrm{~km}$ is preferable to a decrement by a same amount. Apparently there is no justification in studying EoL disposal by eccentricity build-up considering an initial decrement in the spacecraft semi-major axis (e.g., Alessi et al. (2016) and related works assume a decrement in the initial $a$ by $-1514 \mathrm{~km}$ ). Unavoidably, for very high $\Delta v$, increasing the eccentricity by a reduction of the perigee becomes optimal, being the direct reentry the extremal case.

The maximum $\dot{e}$ occurs when both $C_{2}$ and $C_{3}$ (the dominating terms at the inclination of interest) terms contribute positively to the derivative. This happens when $2 \omega$ lies in the first or second quadrant, and $2 \omega+\Omega$ belongs to third or fourth quadrant. The ideal condition is $2 \omega=90 \mathrm{deg}(\omega=45$ or -135 deg) and when $2 \omega+\Omega=-90 \mathrm{deg}(\Omega= \pm 180 \mathrm{deg})$. The secular growth in the eccentricity occurs when one of trigonometric arguments in the expression of $\dot{e}$ is almost constant, a condition known as inclination-dependent resonance. When only the secular effect of $J_{2}$ is considered, the averaged rate of change of $\omega$ and $\Omega$ are (Cook, 1962)

$$
\begin{aligned}
\dot{\omega} & =\frac{3}{4} J_{2} n\left(\frac{R}{a}\right)^{2} \frac{5 \cos ^{2} i-1}{\left(1-e^{2}\right)^{2}} \\
\dot{\Omega} & =-\frac{3}{2} J_{2} n\left(\frac{R}{a}\right)^{2} \frac{\cos i}{\left(1-e^{2}\right)^{2}} .
\end{aligned}
$$

Thus, $2 \omega$ is constant at the critical inclination of 63.43 deg when $\Omega$ drifts, e.g. for $a=31330 \mathrm{~km}$ and $e=0.0552$ obtained by applying a $\Delta v \approx 100$ $\mathrm{m} / \mathrm{s}$, at a rate of $-0.0171 \mathrm{deg} /$ day $(2 \omega+\Omega$ has a period of 57.64 years $)$, and $2 \omega+\Omega$ is constant at $56.06 \mathrm{deg}$ when $\omega$ drifts at a rate of $0.0107 \mathrm{deg} /$ day and $\Omega$ at a rate of $-0.0214 \mathrm{deg} / \mathrm{day}$ ( $2 \omega$ has a period of 46.24 years). The terms associated with $2 \omega-\Omega$ and $\omega+\Omega$ are typically much smaller and they are in resonance at inclinations of 69 and 46.38 deg respectively, which are outside the range achievable by the disposal manoeuvre and rarely reached during the disposal phase. Having either $2 \omega+\Omega$ or $2 \omega$ close to the resonance condition implies that the other one follows an oscillatory behaviour. This is shown by Fig. 9, in which contour lines of the eccentricity derivative are plotted as function of $\omega$ and $\Omega$ using Eq. (1), for $i=56.06 \mathrm{deg}$ and in panel (a) and for $i=63.43 \mathrm{deg}$ in panel (b). Using Eq. (3) the spacecraft moves 
on this plane on a line with slope given by

$$
\frac{\mathrm{d} \Omega}{\mathrm{d} \omega}=-2 \frac{\cos i}{5 \cos ^{2} i-1} .
$$

For example, the spacecraft moves on vertical lines at the critical inclination $i=63.43 \mathrm{deg}$, (as shown in Fig. 9(b)), and on lines with slope -2 when $i=56.06 \mathrm{deg}$ (as shown in Fig. 9(a)). In particular, red lines highlight the conditions for which the contributions of $C_{3} \sin (2 \omega+\Omega)$ and $C_{2} \sin 2 \omega$ to the eccentricity growth are maximum, and blue lines the conditions for which the contributions to the derivative of the eccentricity are minimum. Note that at critical inclination, Fig. 9(b), the spacecraft will move along vertical lines from top to bottom as $\dot{\omega}=0$ and $\dot{\Omega}<0$. For $i=56.06 \mathrm{deg}$, in Fig. 9(a), the spacecraft will move on the $2 \omega+\Omega=$ const lines from top to bottom, as $\dot{\omega}>0$ and $\dot{\Omega}<0$. As we will see later the inclination of the spacecraft will vary during the disposal, thus the actual trajectory is not a straight line in the $\Omega-\omega$ plane. At the Galileo inclination, $2 \omega+\Omega \approx$ const (i.e., close to resonant condition) and the spacecraft moves in the $\Omega-\omega$ plane with a slope close to -2 . To achieve a fast reentry, a secular growth of the eccentricity is needed; thus, the disposal manoeuvre needs to move the initial condition close to a red line. This result explains well why for a given initial value of $\Omega$ there is a range of $\omega$ that produces high eccentricity growth and a range of $\omega$ that is characterised by small increase of the eccentricity. As the initial $\Omega$ cannot be largely changed due to $\Delta v$ requirements, for a given initial $\Omega$ this is achieved by a change of the $\omega$ such that $2 \omega+\Omega \approx-90 \mathrm{deg}$. However, even along red lines, there are always phases in which the eccentricity decreases (in Fig. 9(a) for $\Omega \in[-42,42] \mathrm{deg}$ ) due to the contribution of the $C_{2} \sin 2 \omega$ term. Thus, the optimal values for the initial $\Omega$ are those for which the motion of the spacecraft starts right at the beginning of the phase in which $\dot{e}>0$, i.e. in the region $\Omega \approx 320 \mathrm{deg}$. This allows the amplitude of the $C_{3}$ to grow fast, resulting in a fast reentry disposal. In summary, although the secular growth of the eccentricity is a result of the $2: 1$ resonance, the term $C_{2} \sin 2 \omega$ is equally important in determining the eccentricity evolution. Note that is the $C_{2} \sin 2 \omega$ term that is responsible of the curls in panels (b) of Fig. 2-4. This is a consideration that cannot be found in the reviewed literature as researchers have been paying attention almost exclusively at resonant terms and their overlap, but neglecting the contribution of non-resonant terms (however, authors have already recognised that the 2:1 resonance alone cannot produce reentry trajectories (Rosengren et al., 2017)). As a last remark note that, 
for an initial inclination close to the critical one (not a case for Galileo), the spacecraft moves along almost vertical lines; the region of eccentricity decrease (as shown by Fig. 9(b)) can then be avoided and a much faster reentry is possible.

In Sec. 6 it was shown by numerical experiments that the eccentricity growth is largely affected by the configuration of the Moon. At Galileo's inclination the Moon plays a key role in determining the eccentricity derivatives, in particular in delimiting the regions in which $\dot{e}>0$ from those in which $\dot{e}<0$. Figure 10 shows how the $\dot{e}$ is affected by the Moon's inclination. It can be appreciated that when the Moon's inclination is maximum the range of $\Omega$ in which $\dot{e}<0$ reduces to $[-36.7,36.7]$ deg, whereas it increases to $[-48.1,48.1]$ deg when Moon's inclination is minimum. This is in agreement with the dependance of the amplitudes $K_{i}$ on Moon's inclination already illustrated in Fig. 8. Passing through regions of low $\Omega$ when the Moon's inclination is maximum represents an optimal condition, as this allows for a limited decrease of the eccentricity.

The effect of the Moon's configuration is studied by analysing the maximum eccentricity reached in 100 years for different initial conditions and epochs, using the double-averaged SA propagator $(100 \times 100$ grid on $\Omega$ and $\omega)$. In Fig. 11 contour lines are plotted for increments of 0.1 in eccentricity. First of all note that, in agreement with the literature (see for example Merguizo Sanchez et al. (2015); Alessi et al. (2016); Rosengren et al. (2017)), these plots are $180 \mathrm{deg}$ periodic in $\omega$, as one would expected from Eq. 1. In addition, these figures confirm that the eccentricity growth is mainly due to the $2 \omega+\Omega$ resonance, as the regions of maximum eccentricity growth are well aligned with the red lines, and those with minimum increase with the blue lines. Moreover, high initial values of $\Omega$ result in larger eccentricity growth, independently from the Moon's configuration. This is because, as explained by Fig. 9(a), 10(a), and 10(b), the spacecraft will experience an increase of the eccentricity for an extended period of time. Significant eccentricity growths are possible also at lower initial values of $\Omega$, but the maximum growth in these cases are always lower when compared with that achieved with high initial $\Omega$.

The impact of the Moon's configuration on eccentricity growth is always significant. In particular, regions with high eccentricity growth are associated with a passage from regions of eccentricity decrease with a favourable configuration of the Moon, i.e., for high values of $i_{M}$ such that the amplitude $C_{2}$ is decreased and $C_{3}$ is increased. Initially, the spacecraft moves at a rate 
$\dot{\Omega} \approx-0.0214 \mathrm{deg} /$ day, thus covering in a Moon nodal period a $\Delta \Omega \approx 145 \mathrm{deg}$ in the $\omega-\Omega$ plane. This is fully reflected by the behaviour of the eccentricity growth in Fig. 11. For example, in Fig. 11(a) an island of relatively high eccentricity growth is visible for low values of $\Omega$. These initial conditions pass through the region of $\dot{e}<0$ at favourable Moon's inclination: when $\Omega \approx 0$ deg and $i_{M} \approx 28.72 \mathrm{deg}$, thus the initial eccentricity decrease is minimised. The next islands of relative high eccentricity growth in the same plot are placed at initial $\Omega \approx 145 \mathrm{deg}$ higher than the previous ones. This is because initial conditions on these islands encounter the Moon in a similar configuration during their passage at $\dot{e}<0$ (remember that the spacecraft covers approximately $\Delta \Omega \approx 145 \mathrm{deg}$ in a Moon nodal period). For the same reason, Fig. 11(b) shows that regions with low initial values of $\Omega$ can result in high eccentricity growth when the initial $i_{M}=28.72 \mathrm{deg}$. The island of relatively high eccentricity growth around $\Omega \approx 100 \mathrm{deg}$ in Fig. 11(c) is explained by the fact that, when the initial $i_{M}$ has a mean value but with $\mathrm{d} i_{M} / \mathrm{d} t<0$, it takes approximately 13.95 years to reach the maximum, a time in which the spacecraft covers $\Delta \Omega \approx 108 \mathrm{deg}$. A similar reasoning explains the island around $\Omega \approx 72 \mathrm{deg}$ in Fig. $11(\mathrm{~d})$ for $i_{M}=18.14 \mathrm{deg}$. Finally, note that the initial $i_{M}$ plays a key role in strengthening or weakening the maximum eccentricity growth that can be achieved for high initial values of $\Omega$. In particular, $18.14<i_{M}<23.43 \mathrm{deg}$ results in a magnification of the eccentricity growth as shown by Fig. 11(a) and 11(d).

The key role played by the Moon has now been fully clarified. However, we haven't commented on the role played by the Moon's node that appears explicitly in the arguments of Eq. (1) (recall that the Sun's node can be assumed equal to zero). At the optimal value of the resonant angle $2 \omega+\Delta \Omega \approx$ $-90 \mathrm{deg}$, the change of $\Omega_{M}$ has a maximum effect on the total $\dot{e}$ of about $3 \%$. Of course this effect can increase for different values of the resonance angle, but this is of secondary importance with respect to the Moon's inclination, in particular due to our specific interest in eccentricity growth scenarios.

The role played by the initial $a, e, i, \Omega$, and $\omega$ and the Moon's configuration have now been explained. However, pertaining to the spacecraft inclination we have just said that at the Galileo inclination $2 \omega+\Omega \approx$ const, i.e. we are close to a resonance. As this resonance is inclination-dependent, the evolution of the inclination of the spacecraft during the disposal is important to maintain $2 \omega+\Omega \approx$ const during the entire disposal. For low eccentricities (as those at the beginning of the disposal) the variation of the inclination 
can be approximated, using the same notation of Eq. 1, as (Chao, 1998)

$$
\mathrm{d} i / \mathrm{d} t=\frac{3}{8} \frac{n_{3}^{2}}{n} R_{m}\left(\cos i \sin i_{3} \sin \Delta \Omega+\sin i \sin ^{2} i_{3} \sin 2 \Delta \Omega\right) .
$$

The behaviour of the derivative of the inclination is plotted in Fig. 12, where obviously there is no dependance on $\omega$. The inclination increases for $\Omega$ in the first and second quadrant, and decreases in the third and fourth quadrant. This behaviour helps us understanding the effect of the initial inclination shown in Fig. 13, which is based on 100-year propagations obtained with the double-averaged dynamical model for initial inclination of 55.06 and 57.06 deg (results in agreement with Fig. 1 in Rosengren et al. (2017) in which 200-year propagations are considered). Comparing Fig. 13(a) with Fig. 11(a) it can be noticed that the eccentricity growth for central values of initial $\Omega$ is strengthened. This is because in these regions the spacecraft experiences an initial increase in the inclination (remember that the spacecraft moves in the $\omega-\Omega$ plane with an initial rate $\dot{\Omega} \approx-0.0214 \mathrm{deg} /$ day) that allows for a better exploitation of the $2 \omega+\Omega$ resonance. In contrast, an initial node close to zero results in a further initial decrement of inclination, thus moving the spacecraft away from $2 \omega+\Omega$ resonance. Figure 13(b) shows that the eccentricity growth for central initial values of $\Omega$ is reduced when the initial inclination is increased to $57.06 \mathrm{deg}$. The initial increase of the inclination in this case moves the spacecraft away from the resonant inclination of 56.06 deg. In contrast, with an initial node close to zero the spacecraft initially experiences a reduction of inclination, moving towards the $2 \omega+\Omega$ resonance.

\section{Disposal maps}

The analyses provided in the previous section are now used to explain the results obtained by the optimisations. To explain some key elements of the eccentricity growth scenario, disposal maps are plotted in panel (a) and (b) of Fig. 14-19 for solutions with either minimum $\Delta v$ or time-to-reentry. A disposal map is a two-dimensional plot rich in information. The disposal trajectory is plotted in the $\omega-\Omega$ plane. The trajectory is represented by a red line with colour proportional to the eccentricity (the line is darker at higher eccentricities) and width proportional to the inclination (a ticker is used at higher inclinations). A white circular thick is showed every 5 years to appreciate the time evolution of the orbital parameters. Thus, with just a 
two-dimensional line the five relevant information $(\omega, \Omega, e, i, t)$ of the trajectory are depicted (note that the averaged value of semi-major axis is constant and the fast angle is irrelevant in the time scales of interest). The initial conditions at disposal are represented by a red diamond, and the dashed line indicates the disposal manoeuvre. The trajectory line is plotted over the contour levels of $\dot{e}$ (units [1/day]) computed with Eq. 1. As $\dot{e}$ depends on the instantaneous values of both spacecraft and third body parameters, averaged values over the entire disposal phase are used in panels (a). However, during the disposal the large variations of the eccentricity significantly affect the absolute value of $\dot{e}$ (through the amplitudes $C_{i}$ ); thus, to highlight this aspect another version of the disposal map is provided in panels (b), in which the contour levels of $\dot{e}$ are plotted for the instantaneous values of orbital parameters, except for $\omega$ that is varied in the range $[-180,180]$ deg. Figures 14-19 are completed by panels (c) and (d), which provide the evolution of the eccentricity and its derivative (total and the contributions of different terms $C_{i}$, where the contributions from the Sun and the Moon are summed), respectively.

First we analyse the solution with the minimum $\Delta v$, i.e., Fig. 14-16. In all the cases the spacecraft moves close to the 2:1 resonance (slope -2) and the initial conditions are selected such that the passage above the region of positive $\dot{e}$, the white hills, are maximised. When a passage on a white hill occurs then $e$ grows significantly and the trajectory line gets darker. In addition, at higher eccentricities the separation between the time ticks gets larger, in agreement with the secular time derivative of $\omega$ and $\Omega$ according to Eq. (3). The eccentricity growth is always obtained by an interplay of the $C_{2}$ and $C_{3}$ terms. While the $2: 1$ resonance is the main source of the secular growth of $e$ (its contribution is positive for the entire length of the disposal), the superimposed oscillatory behaviour is due to the $2 \omega$ term. When both terms are positive (determining the white hills) a steep increase in the eccentricity is achieved, and when the $C_{2}$ term is negative the eccentricity derivative can become negative as well. The maximum value of $\dot{e}$ is reached when the eccentricity gets close to 0.71 , i.e., when the $e 1-e^{2}$ term reaches its maximum. As already pointed out in Sec. 8, SSC 37846 (i.e., the spacecraft with highest $\Delta v$ demand) is the only one for which the initial eccentricity decreases before taking the eccentricity growth path. For Sol \#1 this occurs after the eccentricity reaches a minimum value close to 0.08 , which is comparable with the initial eccentricity of SSC 41175 and 40890. For SSC 40890 the initial eccentricity is the lowest, $e=0.048$, and this explains 
the lowest value the disposal $\Delta v$. The different required initial eccentricities are due to both the different initial $\Omega$ of the spacecraft and the fact that a disposal manoeuvre cannot significantly change this angle due to high $\Delta v$ requirements (indeed in all the disposal maps the manoeuvre doesn't change $\Omega$ significantly). The initial value of $\Omega$ for SSC 37846 is very unfavourable, because, independently from the change in $\omega$, the trajectory needs to pass through a region with negative $\dot{e}$, before passing on a white hill. As a result, the disposal manoeuvre must increase significantly the initial eccentricity to enable its long-term increase within 100 years, despite of the unavoidable initial decrease (as confirmed by Fig. 14(c)). The situation is exactly the opposite for SSC 40890: a suitable change of the initial $\omega$ places the spacecraft right at the beginning of a white hill, thus allowing for a long-term reentry with minimum propellant consumption (and minimum required variation of e). SSC 41175 is in a intermediate condition: a high initial eccentricity growth can be obtained by moving the initial $\omega$ on the top of a white region, but the initial build-up is lower compared to SSC 40890. Thus, a slightly higher increase in the initial eccentricity is required to reenter within 100 years. Note that a possible strategy to minimise the disposal $\Delta v$ would be to wait until $\Omega$ naturally reaches a favourable value. For Galileo spacecraft the natural drift of $\Omega$ is of the order of $10 \mathrm{deg} /$ year, unfortunately making this strategy not viable when large variations of initial $\Omega$ are necessary.

The initial epoch for the disposal corresponds to the minimum value of $i_{M}$. With the support of Fig. 11(d) we can see that this is close to an optimal condition for the initial $\Omega$ of both SSC 37846 and SSC 40890, explaining why in the optimisation with free initial epoch the disposal $\Delta v$ could not be further reduced. On the other hand, the results of the optimisation with free initial epoch illustrated in Sec. 6 shows that the disposal $\Delta v$ is significantly reduced for SSC 41175, when the epoch corresponding to $i_{M}=21.6 \mathrm{deg}$ and $\mathrm{d} i_{M} / \mathrm{d} t>0$ is selected. This allows for a limitation of the eccentricity decrease when the spacecraft encounters for the first time the region in which $\dot{e}<0$.

Looking at Fig. 2(e), 3(e), and 4(e) it can be appreciated that the solutions with minimum $\Delta v$ are characterised by initial inclination close to 56.06 for SSC 37876, a higher value for SSC 40890, and a lower value for SSC 41175. This result is fully aligned with the analysis proposed in the previous section regarding the initial inclination. In particular, the initial node of SSC 41175 puts the spacecraft close to a region of large inclination increase, thus an initial low value of the inclination is required (as shown by Fig. 13(a)). The 
opposite condition occurs for SSC 40890, thus a initial high value of the inclination is selected to optimise the eccentricity build-up (as shown by Fig. 13(b)).

The situation is quite different when short-term (high $\Delta v$ ) reentries are analysed. For SSC 37846 the manoeuvre moves the initial inclination closer to the critical inclination (see Fig. 2(e)) and the initial phase of the eccentricity growth is determined by the $C_{2}$ term. After the inclination decrease associated to the passage in the region $180<\Omega<360 \mathrm{deg}$, the spacecraft switches to the 2:1 resonance, which causes the final reentry (see Fig. 17). For SSC 41175 the $C_{2}$ and $C_{3}$ terms are both close to their maximum values directly at the beginning of the manoeuvre (see Fig. 18) due to the initial value of $\Omega$. Thus, a manoeuvre that increases the initial $a$ to $45317.18 \mathrm{~km}$ and $e$ to 0.347 allows for the quickest reentry in around 12 years. The secular growth of $e$ for SSC 40890 is associated with the 2:1 resonance, but the $C_{2}$ term is of fundamental importance to further increase the eccentricity in the initial phase of the disposal, as shown by Fig 19.

Lastly, it can be noticed that the Pareto fronts of Fig. 1 are discontinuous in the time of reentry. By looking at the disposal maps it can be understood that if one solution does not reach the reentry eccentricity with a pass on a white hill, it will need to wait until the next passage on the $\dot{e}$ hill to actually reenter. This effect can explain the jumps in reentry time that characterise the Pareto fronts.

Note that relying on Eq. (3) the thickness of the lines (proportional to the spacecraft inclination) in all disposal maps should be directly related to the slope of the lines in the $\omega-\Omega$ plane, according to the relation of Eq. (3). In particular, for inclination above the critical inclination the slope should turn positive as both $\dot{\omega}$ and $\dot{\Omega}$ should be negative. A more accurate analysis reveals that this is not always the case. As an example in Fig. 14(a) the thickness is not always proportional to the (negative) slope, and the slope is never positive even above the critical inclination, as it can be appreciated for example by comparing Fig. 17(a) with Fig. 20(b). Figure 20(c) highlights that, when only $J_{2}$ is accounted for, $\dot{\omega}$ reaches a first local minimum after approximately 18 years, while it actually has a local maximum when other perturbations are included. This aspect is fully represented by the disposal map in Fig. 14(a): the slope reaches a local maximum with a maximum line thickness (i.e., maximum inclination) after 10 years. This is even more evident when looking at Fig. 17(a), 20(b), and 20(d). Close to the highest value of the inclination (well above the critical one) the $\dot{\omega}$ reaches a maximum positive value, while 
with the $J_{2}$ approximation one would expect a negative minimum. As a conclusion, we can state that the approximation given by Eq. (3), which is typically adopted in studies on resonances (e.g., Daquin et al. (2016); Celletti et al. (2016); Rosengren et al. (2017)), can be suitable for qualitative analyses, but more complete models shall be utilised in quantitative analyses.

\section{Conclusions}

A method to optimise the reentry EoL disposal for the Galileo constellation was presented. Framing the problem as a multiobjective optimisation one allowed us to find relatively low-cost solutions that enable the spacecraft to reenter into the Earth atmosphere in less than 100 years. Working with the manoeuvre $\Delta v$ allowed us to directly obtain information on disposal feasibility in terms of propellant consumption. In addition, the exploration of the search space with an optimiser results in the further benefit of exploring in detail only those regions of the phase space that are relevant for the disposal. Simulations showed that a double-averaged SA propagator including only the Earth $J_{2-8}$ zonal harmonics and lunisolar perturbations is a suitable model for the quantitative study of the long-term evolution of disposal trajectories. Furthermore, the use of the SA propagators was a key in running large scale simulations with reduced computational times. Simulations with 12,000 single-averaged dynamics took on average 1.4 day on a Mac Mini with $2.6 \mathrm{GHz}$ Intel Core i5 processor and 8GB $1600 \mathrm{MHz}$ DDR3 memory. Simulations with 60,000 runs of the double-averaged took less than 0.6 day on the same machine.

Depending on the initial $\Omega$, relatively low-cost $(<100 \mathrm{~m} / \mathrm{s})$ and fast $(<100$ years) reentry disposals are achievable. With a larger $\Delta v$ budget reentry in less than 20 years is possible, with the additional benefit of minimising onorbit collision risk. These solutions could become feasible only with the use of much more efficient propulsion systems, as those currently under consideration for the second generation of the Galileo constellation.

It was shown that the formula provided by Chao and Gick (2004) for $\dot{e}$ can fully explain the eccentricity build-up scenario. Sun and Moon contributions are of the same order of magnitude, and both these perturbations shall be taken into account. In all cases the eccentricity growth is determined by the combined effect of $C_{3} \sin (2 \omega+\Omega)$ and $C_{2} \sin 2 \omega$ terms in Eq. (1). While the 2:1 resonance is in most cases responsible for the secular growth of the eccentricity, the term $C_{2} \sin 2 \omega$ plays a key role in boosting or slowing down 
the eccentricity increase. Neglecting one of two terms would compromise the full understanding of the eccentricity growth phenomenon. Based on this analysis we have explained which are the optimal values of the initial $\Omega$, and shown how, for a given $\Omega$, the initial $\omega$ should be selected to place the spacecraft on a path of maximum eccentricity growth.

The role played by Moon's configuration was highlighted by sensitivity analyses to disposal epoch as well as with an optimisation with free disposal epoch. It was shown that Moon's inclination is important for the evolution of the eccentricity, and we identified those conditions that promote the eccentricity build-up as a function of the initial $\Omega$.

It is well known that the spacecraft inclination is determinant for exploiting different resonances. With respect to current literature, we were able to explain how the initial inclination should be selected depending on the initial $\Omega$. This was based on the study of the evolution of inclination, explained by Eq. (5) and Fig. 12.

We have proposed a new way of representing the disposal trajectories: the disposal maps. A disposal map is a $2 \mathrm{D}$ plot that fully describes the evolution of all the relevant orbital parameters of the object $(e, i, \Omega, \omega)$ including time information. By means of the disposal maps the key role played by the initial $\Omega$ in the reentry disposal of Galileo spacecraft was clearly shown. This allowed us also to note that the use of $J_{2}$ to represent the secular drift of $\omega$ and $\Omega$ should be limited to qualitative studies.

All the results presented in this work were validated with three different propagators with different level of accuracy. This constitute a hint that, although the dynamics are chaotic, the reentry disposal could be flown without the need of corrective manoeuvres. To back up this statement a rigorous analysis of the sensitivity of the disposal trajectories to realistic orbit determination uncertainties, errors in manoeuvre implementation, and unmodeled dynamics is planned as future work. Future study will also be devoted to the efficient design of graveyard orbits for GNSS.

Overall we have delivered a practical method to design EoL disposals of GNSS spacecraft via eccentricity build-up and to explain the key features of the dynamics, filling in some of the gaps currently present in the literature.

\section{Acknowledgments}

R. Armellin is grateful to Dipen Mistry who implemented the first version of the disposal optimiser and to Mirco Rasotto for setting up the AIDA 
simulations. The authors are grateful to Martn Lara who contributed in the development of the semi-analytical propagators and to David Gondelach for detailed comments on the work. The feedback of the anonymous reviewers contributed significantly to improve the quality of our research. The authors acknowledges the support received by the Marie Sklodowska-Curie grant 627111 (HOPT - Merging Lie perturbation theory and Taylor Differential algebra to address space debris challenges). This work has been funded by the Spanish State Research Agency and the European Regional Development Fund under Project ESP2016-76585-R (AEI/ERDF, EU).

\section{References}

Alessi, E., Rossi, A., Valsecchi, G., Anselmo, L., Pardini, C., Colombo, C., Lewis, H., Daquin, J., Deleflie, F., Vasile, M., Zuiani, F., Merz, K., 2014. Effectiveness of GNSS disposal strategies. Acta Astronautica 99 (Supplement C), $292-302$.

URL http://www.sciencedirect.com/science/article/pii/ S0094576514001052

Alessi, E. M., Deleflie, F., Rosengren, A. J., Rossi, A., Valsecchi, G. B., Daquin, J., Merz, K., May 2016. A numerical investigation on the eccentricity growth of GNSS disposal orbits. Celestial Mechanics and Dynamical Astronomy 125 (1), 71-90.

URL https://doi.org/10.1007/s10569-016-9673-4

Armellin, R., San-Juan, J. F., Lara, M., 2015. End-of-life disposal of high elliptical orbit missions: The case of INTEGRAL. Advances in Space Research 56 (3), 479 - 493, advances in Asteroid and Space Debris Science and Technology - Part 1.

URL http://www.sciencedirect.com/science/article/pii/ S0273117715002227

Breiter, S., Sep 2001. Lunisolar resonances revisited. Celestial Mechanics and Dynamical Astronomy 81 (1), 81-91.

URL https://doi.org/10.1023/A:1013363221377

Celletti, A., Galeç, C., Pucacco, G., 2016. Bifurcation of lunisolar secular resonances for space debris orbits. SIAM Journal on Applied Dynamical Systems 15 (3), 1352-1383.

URL https://doi.org/10.1137/15M1042632 
Celletti, A., Galeç, C. B., 2016. A study of the lunisolar secular resonance $2 \dot{\omega}+\dot{\Omega}=0$. Frontiers in Astronomy and Space Sciences 3, 11 .

URL https://www.frontiersin.org/article/10.3389/fspas.2016. 00011

Chao, C., 2000. MEO disposal orbit stability and direct reentry strategy. In: Advances in the Astronautical Sciences. Vol. 105. pp. 817-837.

Chao, C., Gick, R., 2004. Long-term evolution of navigation satellite orbits: GPS/GLONASS/GALILEO. Advances in Space Research 34 (5), 1221 1226, space Debris.

URL http://www.sciencedirect.com/science/article/pii/ S0273117704001024

Chao, C. C., 1998. Applied orbit perturbation and maintenance. The Aerospace Press. American Institute of Aeronautics and Astronautics, El Segundo, California.

Chao, C. C., Schmitt, D. L., 1990. Eliminating GPS stationkeeping maneuvers by changing the orbit altitude. In: Astrodynamics 1989. pp. 623-643.

Chapront, J., Francou, G., 2003. The lunar theory ELP revisited. Introduction of new planetary perturbations. Astronomy and Astrophysics 404, $735-742$.

Chapront-Touze, M., Chapront, J., 1988. ELP 2000-85 - A semi-analytical lunar ephemeris adequate for historical times. Astronomy and Astrophysics 190, 342-352.

Coello Coello, C. A., Lechuga, M. S., 2002. Mopso: A proposal for multiple objective particle swarm optimization. In: Proceedings of the Evolutionary Computation on 2002. CEC '02. Proceedings of the 2002 Congress - Volume 02. CEC '02. IEEE Computer Society, Washington, DC, USA, pp. 10511056.

URL http://dl.acm.org/citation.cfm?id=1251972.1252327

Cook, G. E., 1962. Luni-solar perturbations of the orbit of an earth satellite. Geophysical Journal of the Royal Astronomical Society 6 (3), 271-291. URL http://dx.doi.org/10.1111/j.1365-246X.1962.tb00351.x 
Daquin, J., Rosengren, A. J., Alessi, E. M., Deleflie, F., Valsecchi, G. B., Rossi, A., Apr 2016. The dynamical structure of the MEO region: longterm stability, chaos, and transport. Celestial Mechanics and Dynamical Astronomy 124 (4), 335-366.

URL https://doi.org/10.1007/s10569-015-9665-9

Deleflie, F., Legendre, P., Exertier, P., Barlier, F., 2005. Long term evolution of the Galileo constellation due to gravitational forces. Advances in Space Research 36 (3), 402 - 411, satellite Dynamics in the Era of Interdisciplinary Space Geodesy.

URL http://www.sciencedirect.com/science/article/pii/ S0273117705005223

Deleflie, F., Rossi, A., Portmann, C., Mtris, G., Barlier, F., 2011. Semianalytical investigations of the long term evolution of the eccentricity of Galileo and GPS-like orbits. Advances in Space Research 47 (5), 811 821, scientific applications of Galileo and other Global Navigation Satellite Systems - II.

URL http://www.sciencedirect.com/science/article/pii/ S0273117710007696

Deprit, A., Mar 1969. Canonical transformations depending on a small parameter. Celestial mechanics 1 (1), 12-30.

URL https://doi.org/10.1007/BF01230629

Gkolias, I., Daquin, J., Gachet, F., Rosengren, A. J., 2016. From order to chaos in earth satellite orbits. The Astronomical Journal 152 (5), 119.

URL http://stacks. iop.org/1538-3881/152/i=5/a=119

Hoots, F. R., Roehrich, R. L., 1980. Models for propagation of NORAD element sets. Tech. rep., DTIC Document.

Hughes, S., 1980. Earth satellite orbits with resonant lunisolar perturbations - I. Resonances dependent only on inclination. Proceedings of the Royal Society of London A: Mathematical, Physical and Engineering Sciences 372 (1749), 243-264.

URL http://rspa.royalsocietypublishing.org/content/372/1749/ 243 
Hughes, S., 1981. Earth satellite orbits with resonant lunisolar perturbations - II. Some resonances dependent on the semi-major axis, eccentricity and inclination. Proceedings of the Royal Society of London A: Mathematical, Physical and Engineering Sciences 375 (1762), 379-396.

URL http://rspa.royalsocietypublishing.org/content/375/1762/ 379

Jenkin, A. B., Gick, R. A., 2002. Collision risk posed to the global positioning system by disposal orbit instability. Journal of Spacecraft and Rockets 39 (4), 532-539.

URL https://doi.org/10.2514/2.3842

Jenkin, A. B., Gick, R. A., Aug. 2005. Dilution of Disposal Orbit Collision for the Medium Earth Orbit Constellations. In: Danesy, D. (Ed.), 4th European Conference on Space Debris. Vol. 587 of ESA Special Publication. pp. 309-314.

Kennedy, J., Eberhart, R. C., 2001. Swarm Intelligence. Morgan Kaufmann Publishers Inc., San Francisco, CA, USA.

Lara, M., San-Juan, J. F., López, L. M., Cefola, P. J., Aug 2012. On the third-body perturbations of high-altitude orbits. Celestial Mechanics and Dynamical Astronomy 113 (4), 435-452.

URL https://doi.org/10.1007/s10569-012-9433-z

Lara, M., San-Juan, J. F., Lpez-Ochoa, L. M., Cefola, P., 2014. Long-term evolution of Galileo operational orbits by canonical perturbation theory. Acta Astronautica 94 (2), 646 - 655.

URL https://www.sciencedirect.com/science/article/pii/ S0094576513003512

Merguizo Sanchez, D., Yokoyama, T., Brasil, P. I. d. O., Cordeiro, R. R., 2010. Some initial conditions for disposed satellites of the systems GPS and Galileo constellations. Mathematical Problems in Engineering 2009, $1-22$.

URL http://dx.doi.org/10.1155/2009/510759

Merguizo Sanchez, D., Yokoyama, T., Prado, A. F. B. d. A., 2015. Study of some strategies for disposal of the GNSS satellites. Mathematical Problems 
in Engineering 2015, 1-14.

URL http://dx.doi.org/10.1155/2015/382340

Morselli, A., Armellin, R., Lizia, P. D., Zazzera, F. B., 2014. A high order method for orbital conjunctions analysis: Sensitivity to initial uncertainties. Advances in Space Research 53 (3), 490 - 508.

URL http://www.sciencedirect.com/science/article/pii/ S0273117713007412

Navarro-Reyes, D., Notarantonio, A., Taini, G., 2009. Galileo constellation: evaluation of station keeping strategies. In: Proceedings 21th International Symposium on Space Flight Dynamics-21th ISSFD. Toulouse, France. pp. $1-13$.

Pardini, C., Anselmo, L., 2012. Post-disposal orbital evolution of satellites and upper stages used by the GPS and GLONASS navigation constellations: The long-term impact on the medium earth orbit environment. Acta Astronautica 77 (Supplement C), 109 - 117.

URL http://www.sciencedirect.com/science/article/pii/ S0094576512000963

Pavlis, N. K., Holmes, S. A., Kenyon, S. C., Factor, J. K., 2012. The development and evaluation of the Earth Gravitational Model 2008 (EGM2008). Journal of Geophysical Research: Solid Earth 117 (B4), n/a-n/a, b04406. URL http://dx.doi.org/10.1029/2011JB008916

Picone, J. M., Hedin, A. E., Drob, D. P., Aikin, A. C., 2002. NRLMSISE-00 empirical model of the atmosphere: Statistical comparisons and scientific issues. Journal of Geophysical Research: Space Physics 107 (A12), SIA 15-1-SIA 15-16, 1468.

URL http://dx.doi.org/10.1029/2002JA009430

Radtke, J., Domnguez-Gonzlez, R., Flegel, S. K., Snchez-Ortiz, N., Merz, K., 2015. Impact of eccentricity build-up and graveyard disposal strategies on MEO navigation constellations. Advances in Space Research 56 (11), $2626-2644$.

URL http://www.sciencedirect.com/science/article/pii/ S0273117715007334 
Rosengren, A. J., Alessi, E. M., Rossi, A., Valsecchi, G. B., 2015. Chaos in navigation satellite orbits caused by the perturbed motion of the Moon. Monthly Notices of the Royal Astronomical Society 449 (4), 3522-3526.

URL http://dx.doi.org/10.1093/mnras/stv534

Rosengren, A. J., Daquin, J., Tsiganis, K., Alessi, E. M., Deleflie, F., Rossi, A., Valsecchi, G. B., 2017. Galileo disposal strategy: stability, chaos and predictability. Monthly Notices of the Royal Astronomical Society 464 (4), 4063-4076.

URL http://dx.doi.org/10.1093/mnras/stw2459

Rossi, A., Apr 2008. Resonant dynamics of medium earth orbits: space debris issues. Celestial Mechanics and Dynamical Astronomy 100 (4), 267-286.

URL https ://doi .org/10.1007/s10569-008-9121-1

Saunders, C. J., Martin, C. E., Lewis, H. G., Aug. 2005. Disposal Orbit Characteristics for Galileo Including Orbit Propagation Techniques. In: Danesy, D. (Ed.), 4th European Conference on Space Debris. Vol. 587 of ESA Special Publication. pp. 685-688.

Standish, E. M., 1998. JPL planetary and lunar ephemerides, DE405/LE405. Jet Propulsion Laboratory Memorandum IOM 312.F-98-048.

Stefanelli, L., Metris, G., 2015. Solar gravitational perturbations on the dynamics of MEO: Increase of the eccentricity due to resonances. Advances in Space Research 55 (7), 1855 - 1867.

URL http://www.sciencedirect.com/science/article/pii/ S0273117715000514 

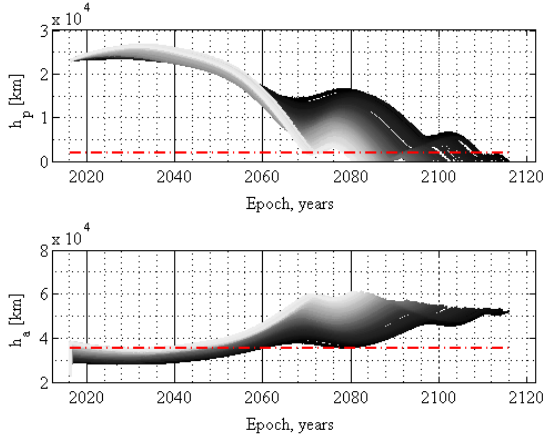

(a) Perigee and apogee profiles

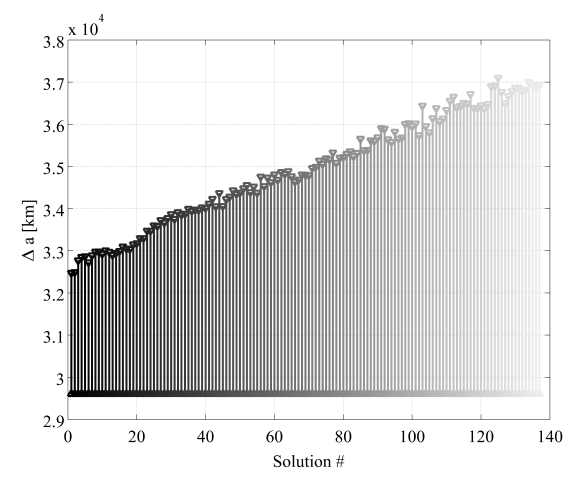

(c) Change in the initial semi-major axis

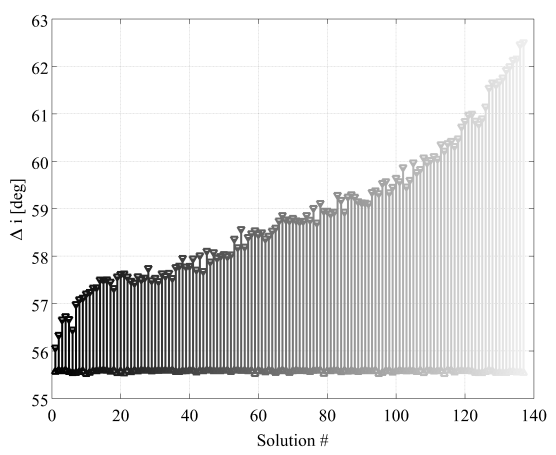

(e) Change in the initial inclination

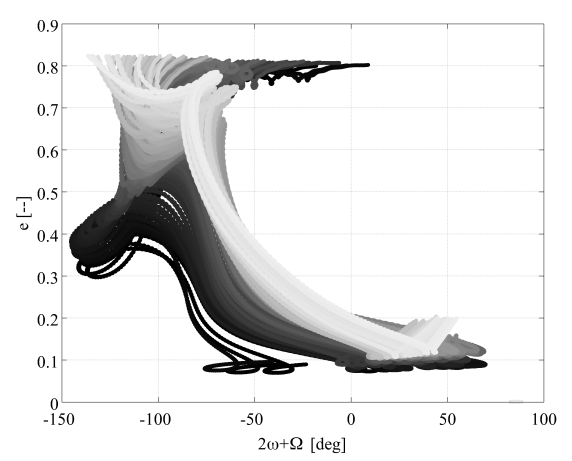

(b) Trajectories in the $e^{-}(2 \omega+\Omega)$ plane

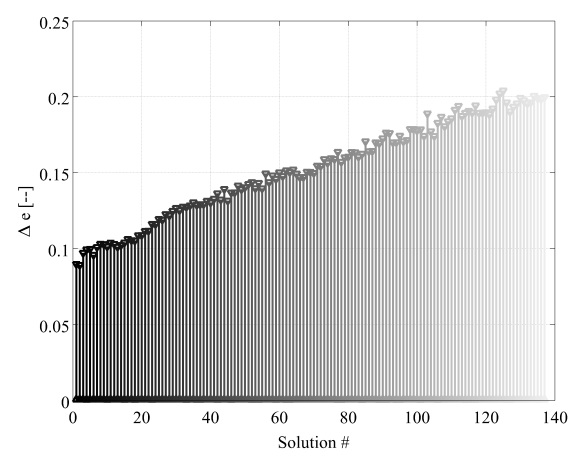

(d) Change in the initial eccentricity

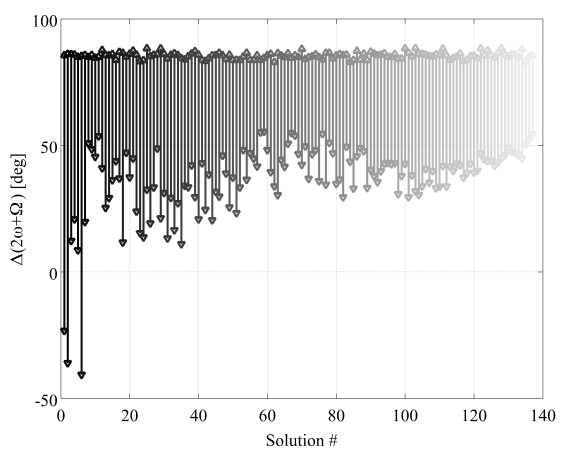

(f) Change in the initial argument of perigee

Figure 2: Pareto optimal solutions for SSC 37846. 

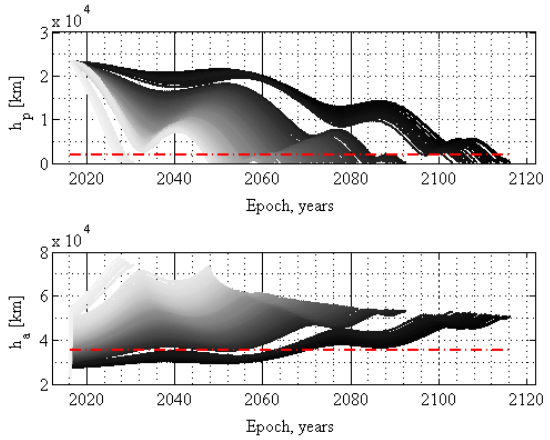

(a) Perigee and apogee profiles

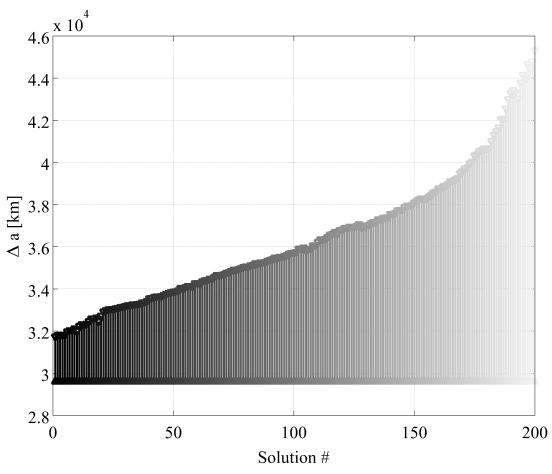

(c) Change in the initial semi-major axis

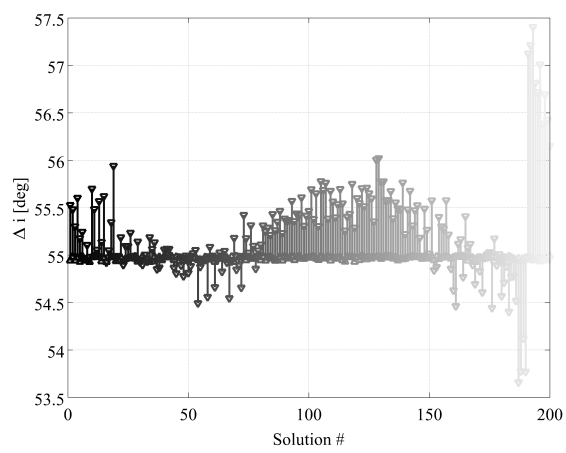

(e) Change in the initial inclination

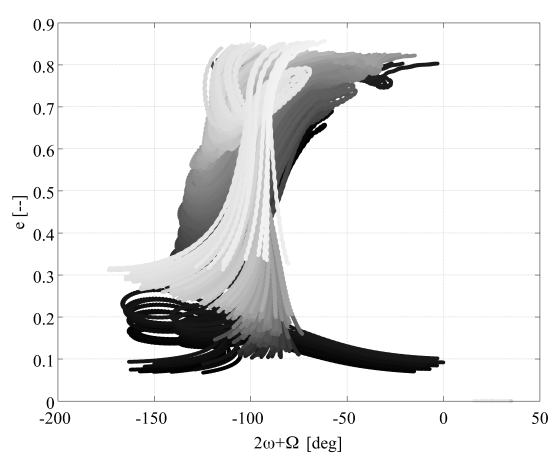

(b) Trajectories in the $e^{-}(2 \omega+\Omega)$ plane

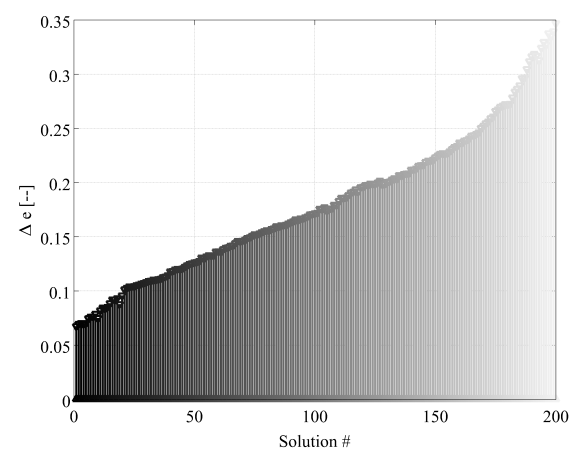

(d) Change in the initial eccentricity

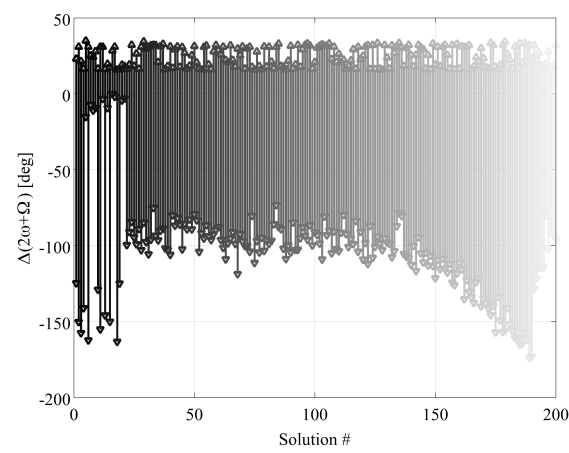

(f) Change in the initial argument of perigee

Figure 3: Pareto optimal solution for SSC 41175. 

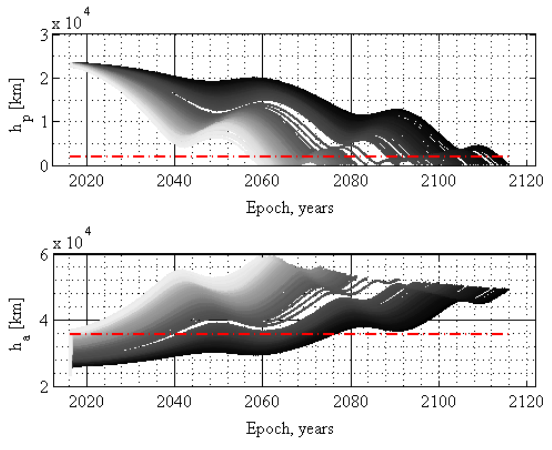

(a) Perigee and apogee profiles

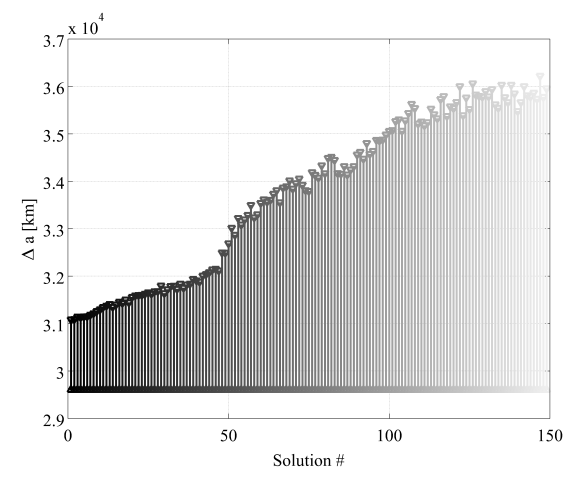

(c) Change in the initial semi-major axis

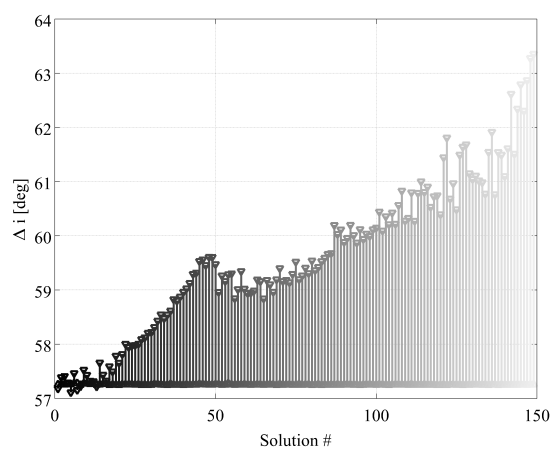

(e) Change in the initial inclination

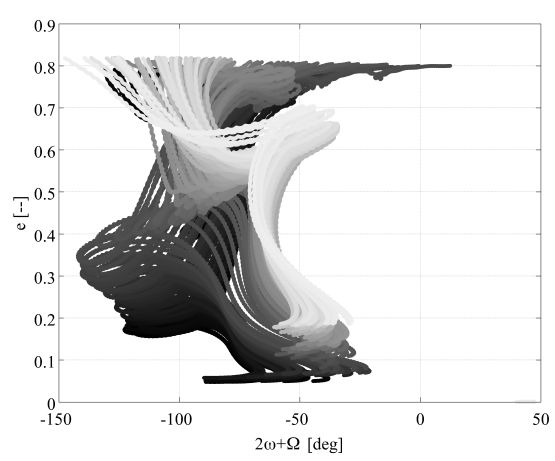

(b) Trajectories in the $e-(2 \omega+\Omega)$ plane

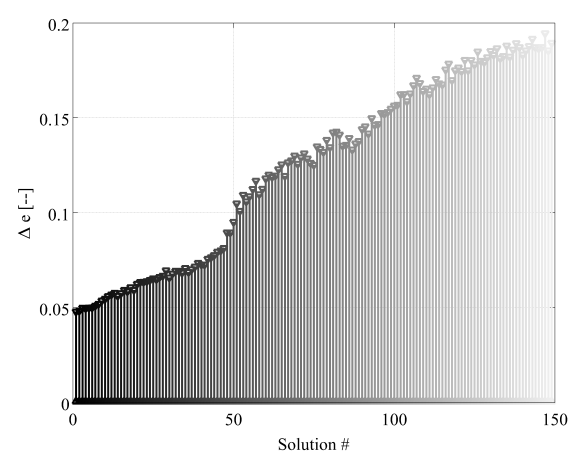

(d) Change in the initial eccentricity

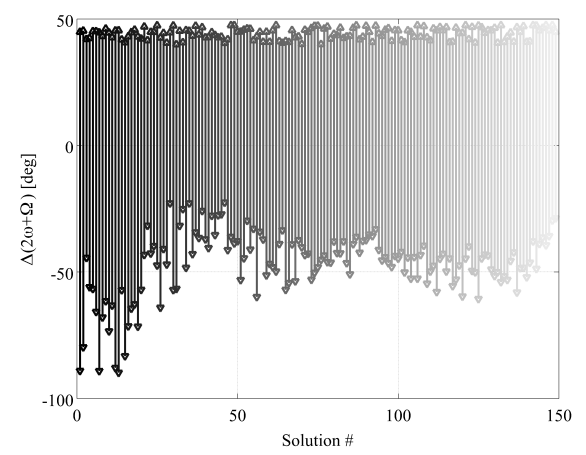

(f) Change in the initial argument of perigee

Figure 4: Pareto optimal solution for SSC 40890. 


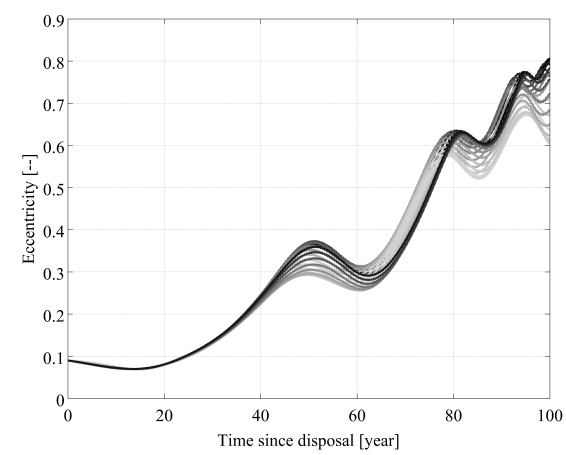

(a) Sensitivity to disposal epoch for SSC 37846, Sol \#1

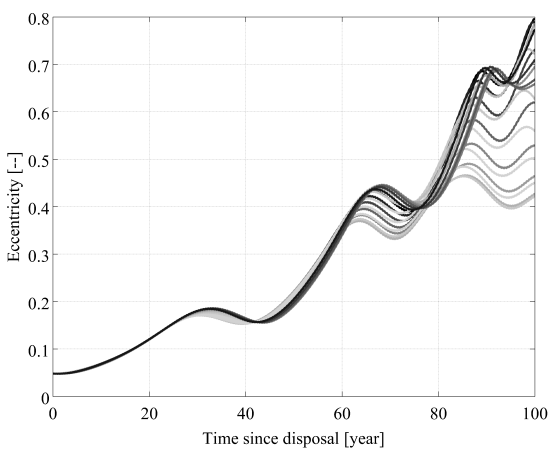

(c) Sensitivity to disposal epoch for SSC 40890, Sol \#1

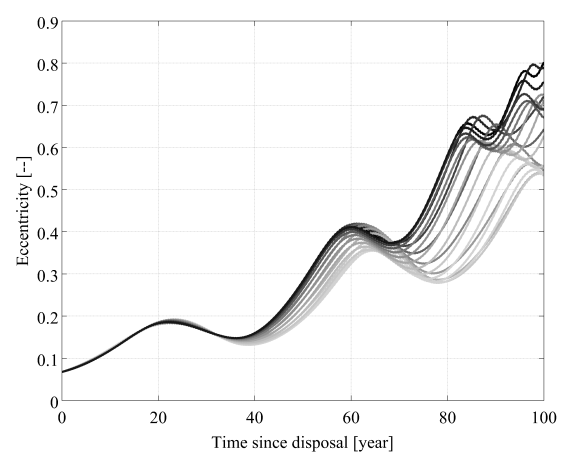

(b) Sensitivity to disposal epoch for SSC 41175, Sol \#1

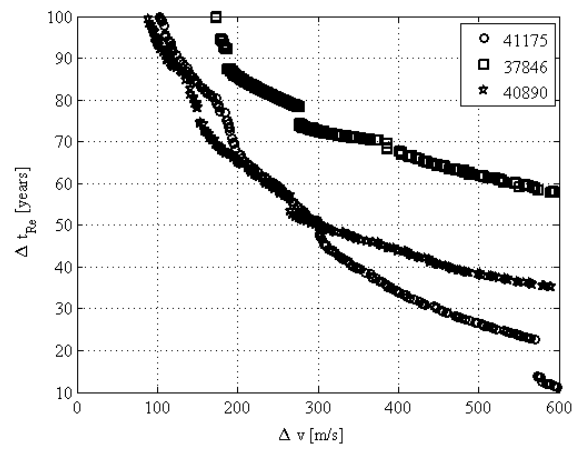

(d) Pareto optimal solution for free disposal epoch

Figure 5: Analysis of the effect of the Moon's configuration. 


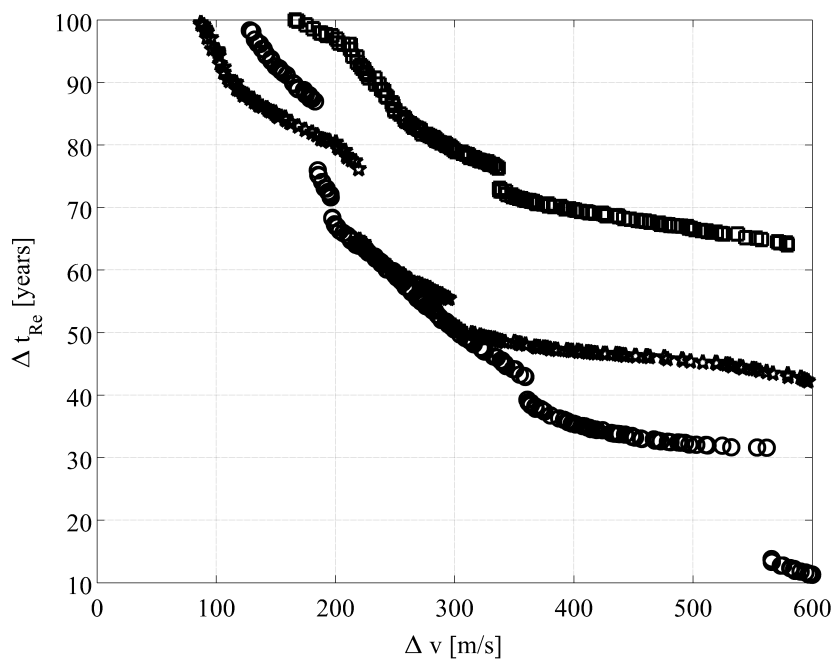

Figure 6: Atmospheric reentry Pareto front obtained with the double-averaged SA propagator.

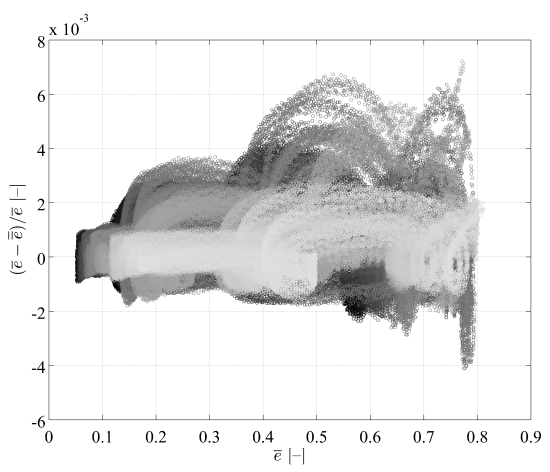

(a) Comparison between SA singleaveraged and SA double-averaged

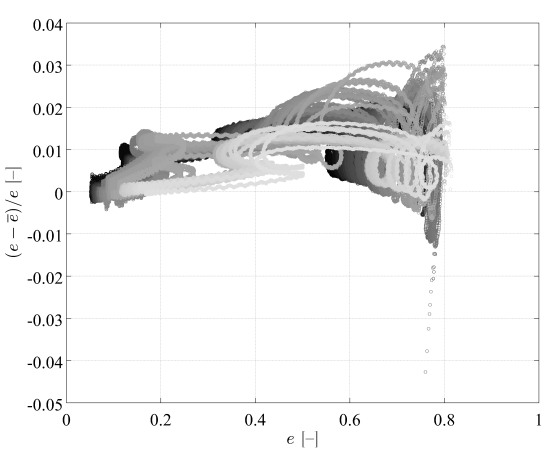

(b) Comparison between AIDA and SA double averaged

Figure 7: Differences in eccentricity evolution. 


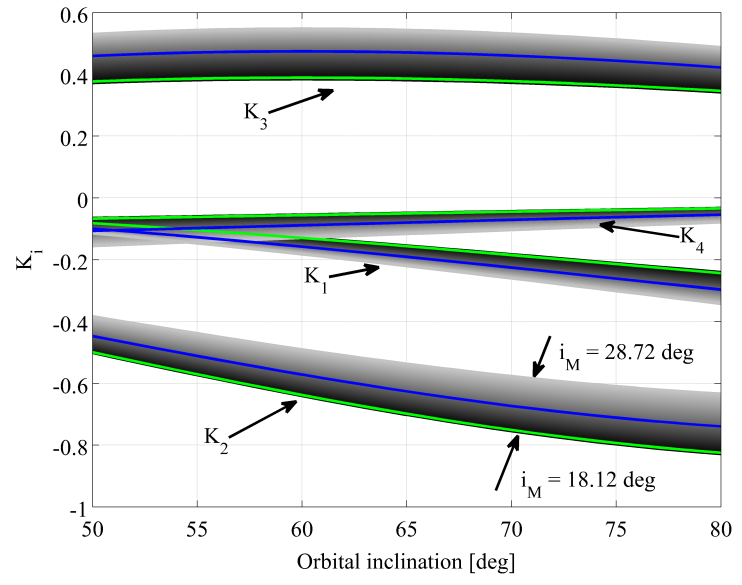

Figure 8: Values of $K_{i}$. The gray scale is used to highlight the variation of the coefficients as a function of the Moon's inclination, darker lines indicate a lower inclination of the Moon. The green line indicates the values of $K_{i}$ corresponding to the inclination of the Moon at the nominal disposal epoch considered in this work. The blue line indicates the $K_{i}$ terms for the inclination of the Sun.

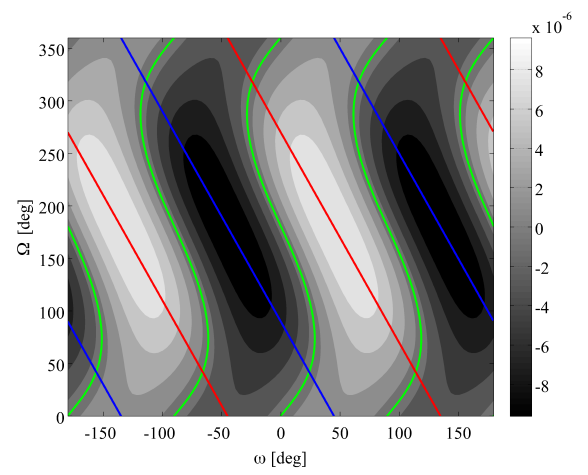

(a) $i=56.06$ deg. The lines indicates condition when $2 \omega+\Omega=$ const, in red when the contribution $C_{3} \sin (2 \omega+\Omega)$ is maximum, and in blue when minimum.

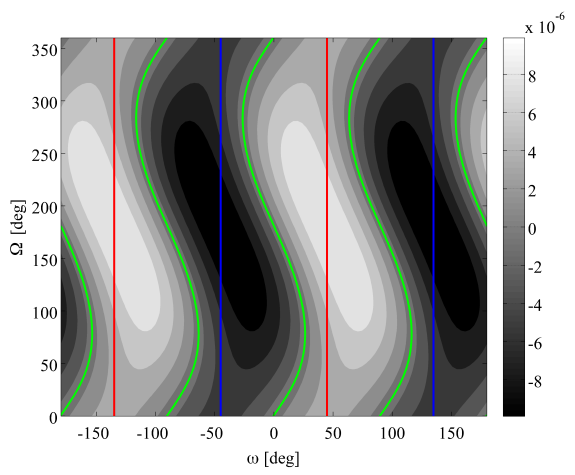

(b) $i=63.43 \mathrm{deg}$. The lines indicates condition when $2 \omega=$ const, in red when the contribution $C_{2} \sin 2 \omega$ is maximum, and in blue when minimum.

Figure 9: Values of the derivative of the eccentricity [1/day] as function of $\omega$ and $\Omega$, for $e=0.552, a=31330 \mathrm{~km}$, and $i_{M}=23.42 \mathrm{deg}$. The green line separates regions where the eccentricity derivative changes sign. 


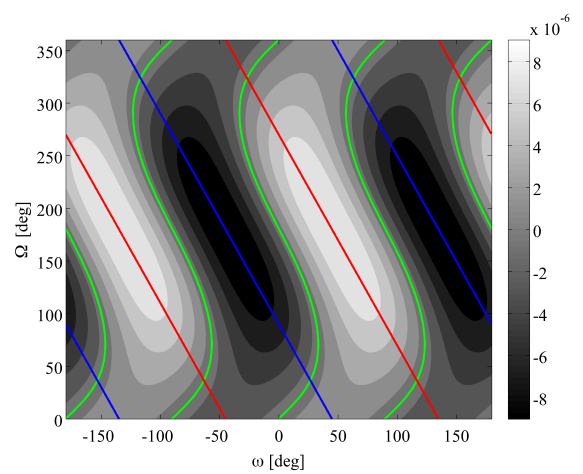

(a) Maximum Moon's inclination $i_{M}=$ $28.72 \mathrm{deg}$

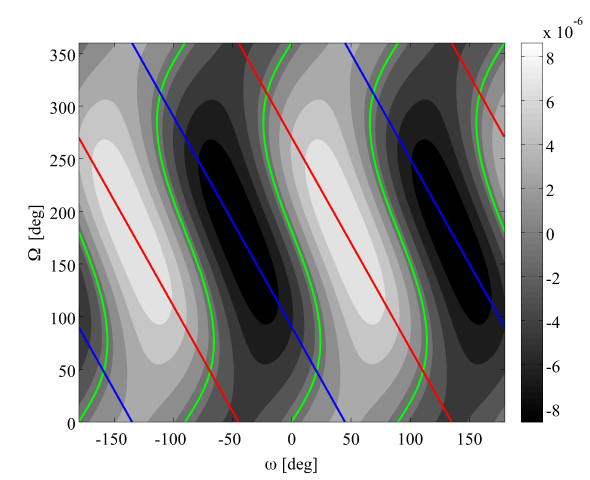

(b) Minimum Moon's inclination $i_{M}=$ $18.14 \mathrm{deg}$

Figure 10: Analysis of the eccentricity derivative [1/day] as function of $\omega$ and $\Omega$, for $i=56.06 \mathrm{deg}, e=0.552, a=31330 \mathrm{~km}$, and different values of the Moon's inclination. The lines indicates condition when $2 \omega+\Omega=$ const, in red when the contribution $C_{3} \sin (2 \omega+\Omega)$ is maximum, and in blue when minimum. The green line separates regions where the eccentricity derivative changes sign. 


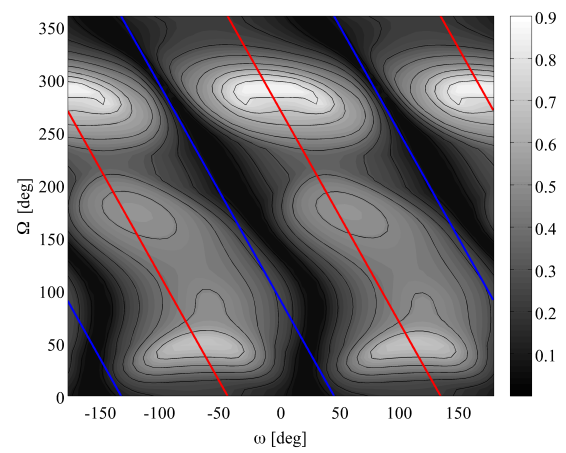

(a) Initial mean Moon's inclination and $\mathrm{d} i_{M} / \mathrm{d} t>0$

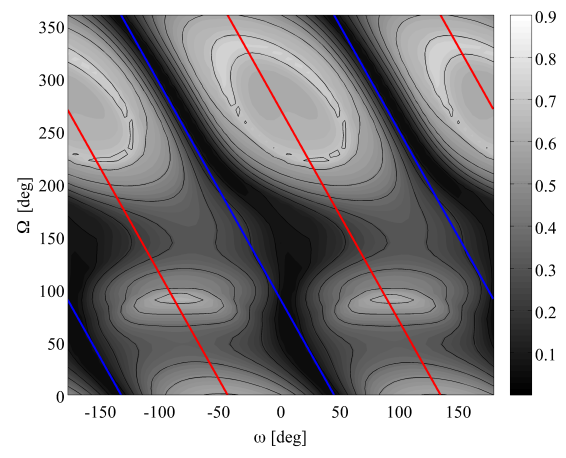

(c) Initial mean Moon's inclination and $\mathrm{d} i_{M} / \mathrm{d} t<0$

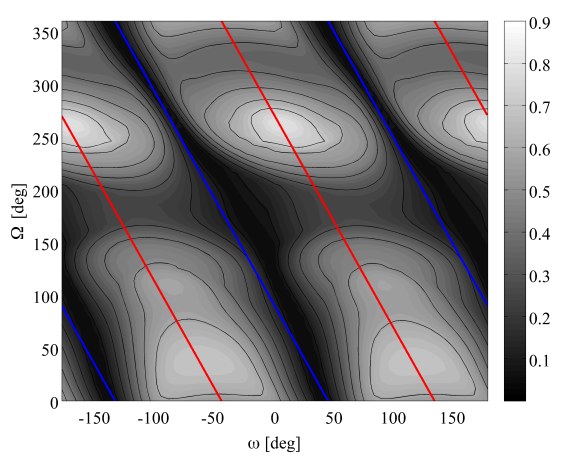

(b) Maximum initial Moon's inclination

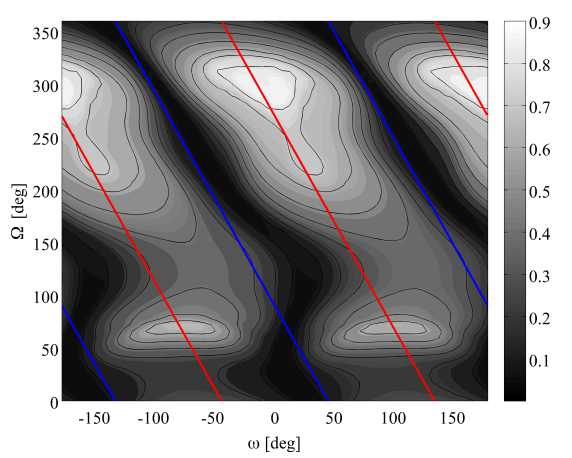

(d) Minimum initial Moon's inclination

Figure 11: Effect of disposal epoch on maximum eccentricity growth for $a=31330 \mathrm{~km}$, $e=0.0552, i=56.06 \mathrm{deg}$. 


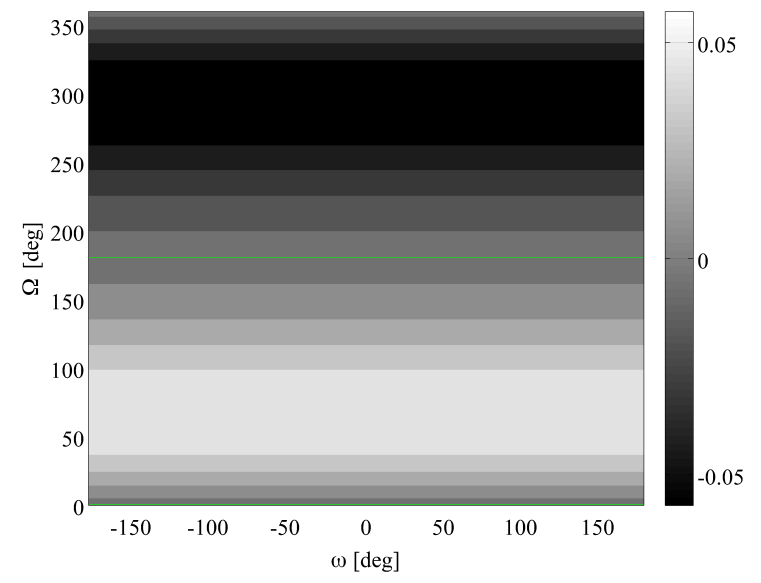

Figure 12: Values of the derivative of the inclination [deg/day] as function of $\omega$ and $\Omega$, for $i=56.06 \mathrm{deg}, i_{M, S}=23.42 \mathrm{deg}$. The green line separates regions where the eccentricity derivative changes sign.

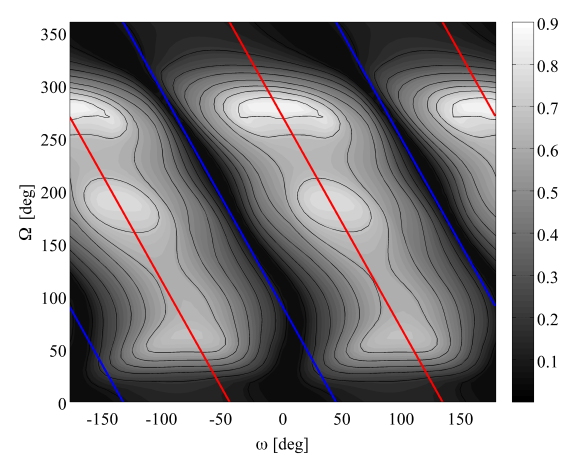

(a) Initial $i=55.06 \mathrm{deg}$

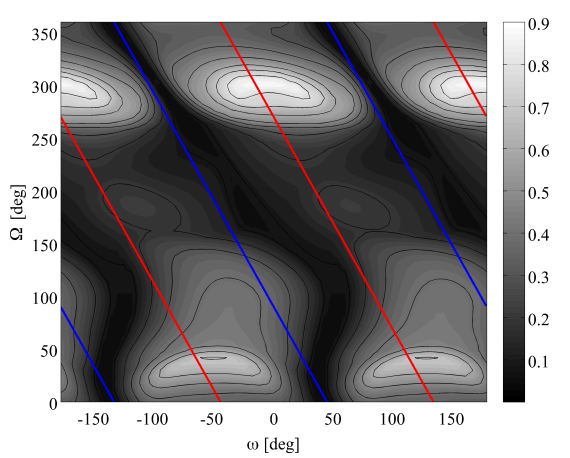

(b) Initial $i=57.06 \mathrm{deg}$

Figure 13: Effect of initial spacecraft inclination on maximum eccentricity growth for $a=31330 \mathrm{~km}, e=0.0552$, initial mean Moon's inclination and $\mathrm{d} i_{M} / \mathrm{d} t>0$. 


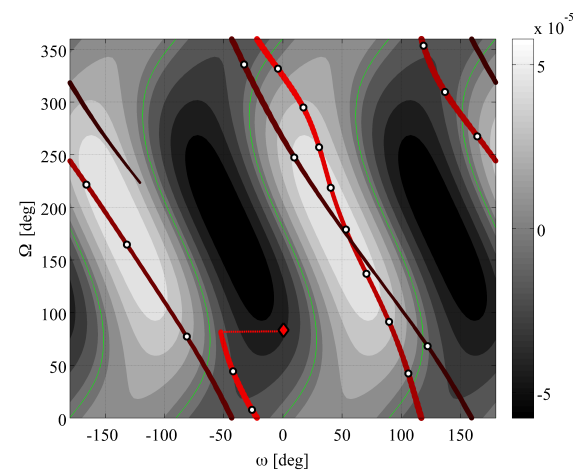

(a) Disposal map for mean orbital elements

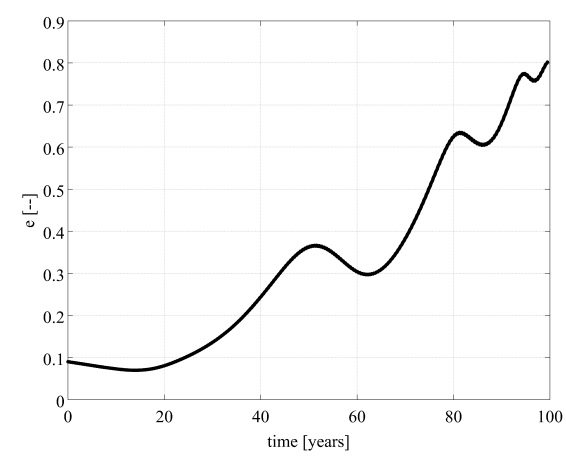

(c) Evolution of eccentricity

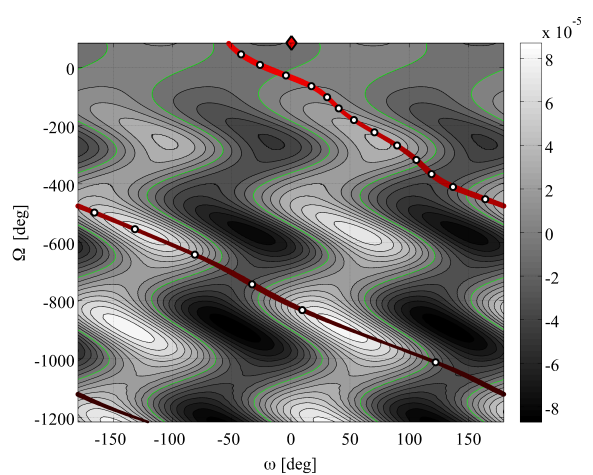

(b) Disposal map for orbital elements evolution

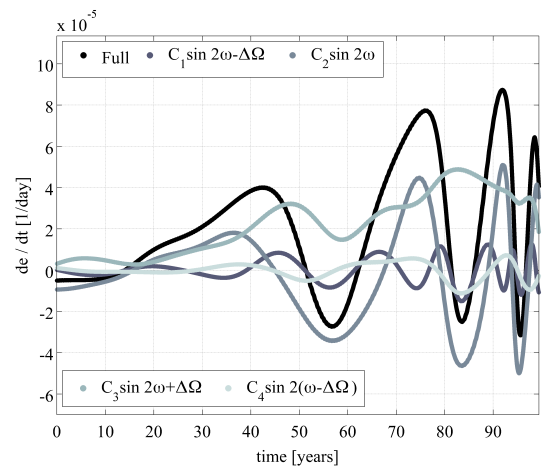

(d) Contribution of different term to eccentricity derivative

Figure 14: Analysis of eccentricity build-up for SSC 37846 and minimum $\Delta v$ (Sol \#1). 


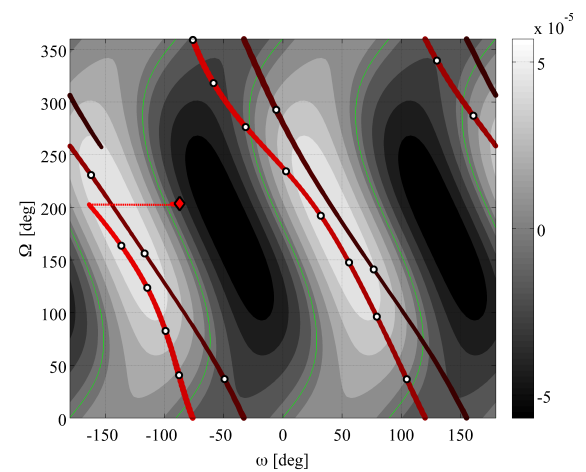

(a) Disposal map for mean orbital elements

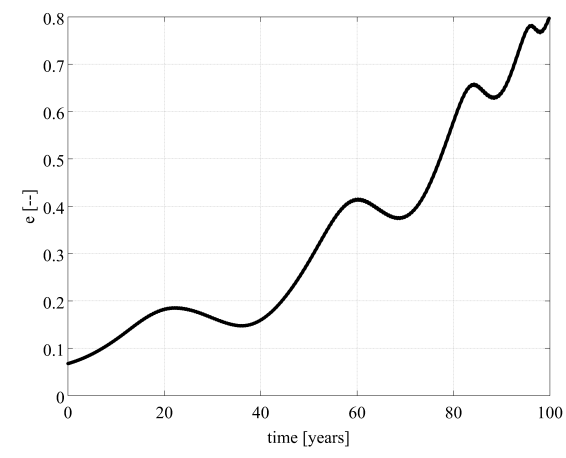

(c) Evolution of eccentricity

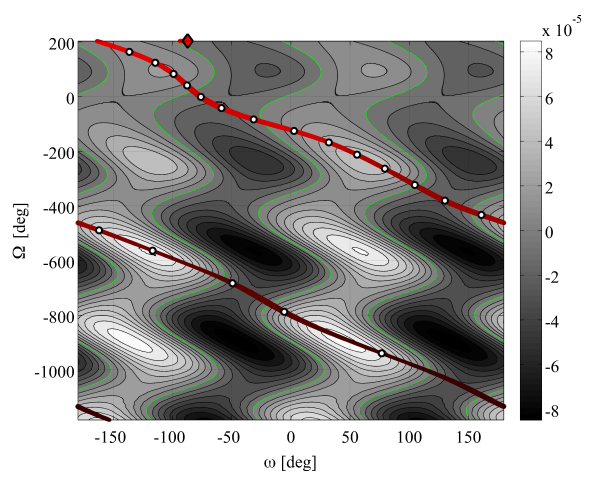

(b) Disposal map for orbital elements evolution

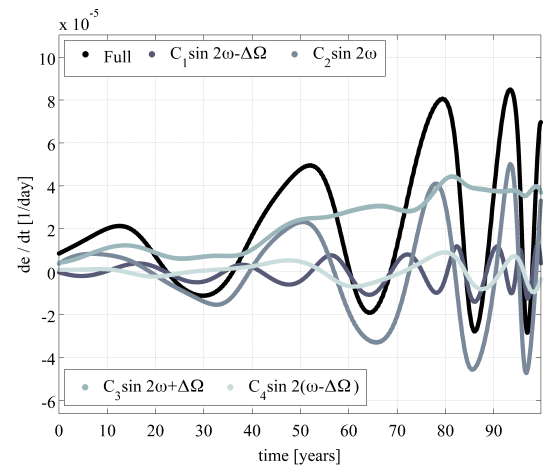

(d) Contribution of different term to eccentricity derivative

Figure 15: Analysis of eccentricity build-up for SSC 41775 and minimum $\Delta v$ (Sol \#1). 


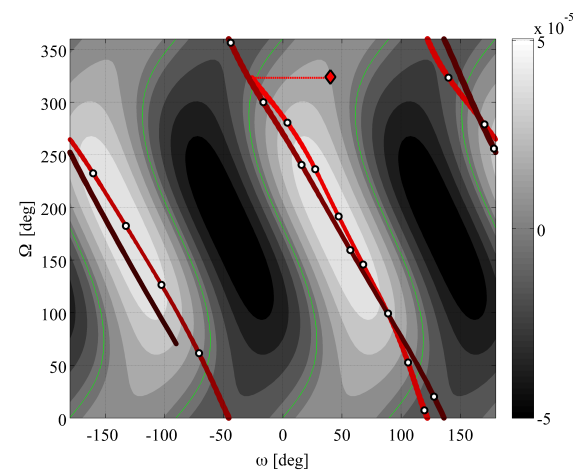

(a) Disposal map for mean orbital elements

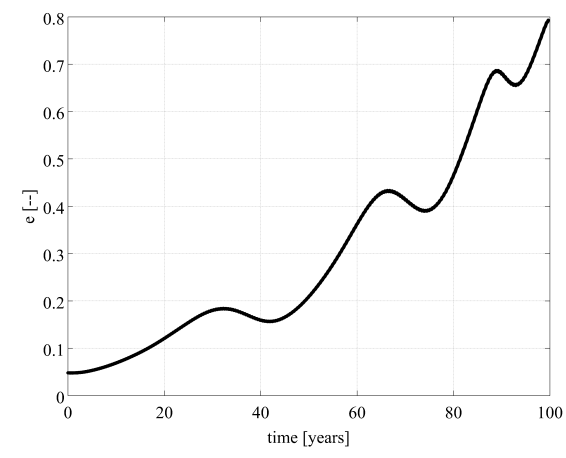

(c) Evolution of eccentricity

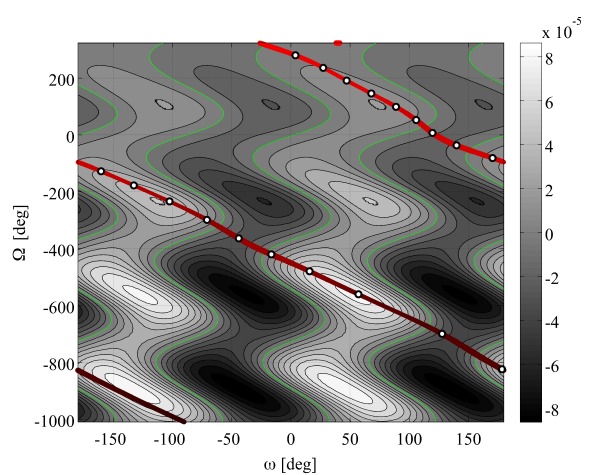

(b) Disposal map for orbital elements evolution

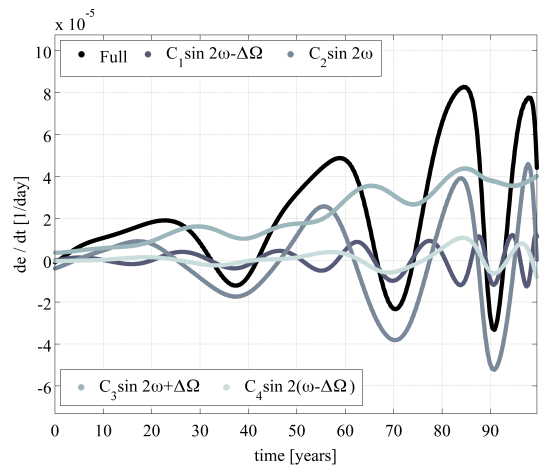

(d) Contribution of different term to eccentricity derivative

Figure 16: Analysis of eccentricity build-up for SSC 40980 and minimum $\Delta v$ (Sol \#1). 


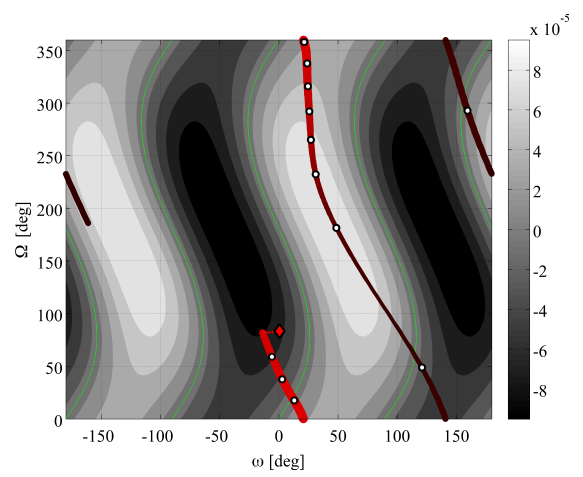

(a) Disposal map for mean orbital elements

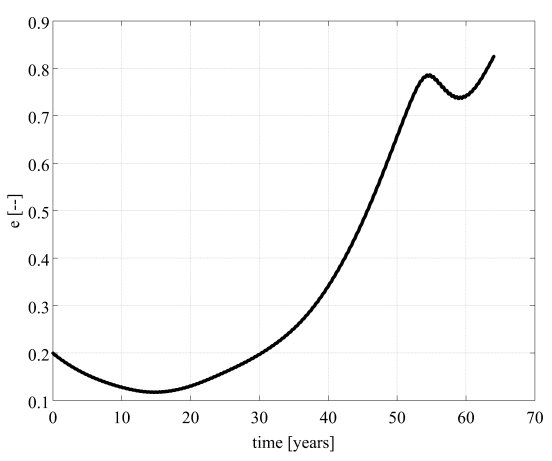

(c) Evolution of eccentricity

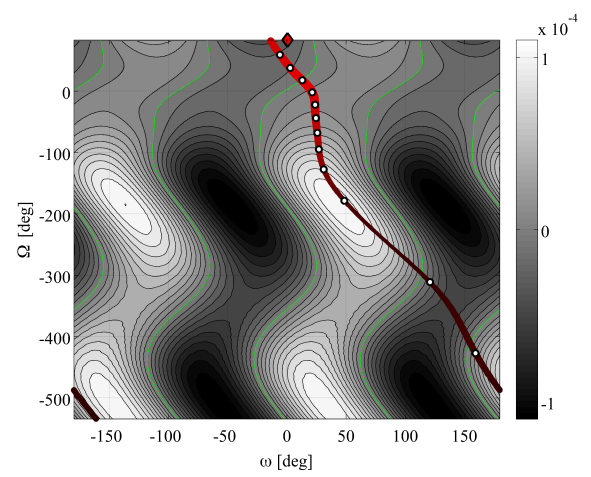

(b) Disposal map for unwrapped node

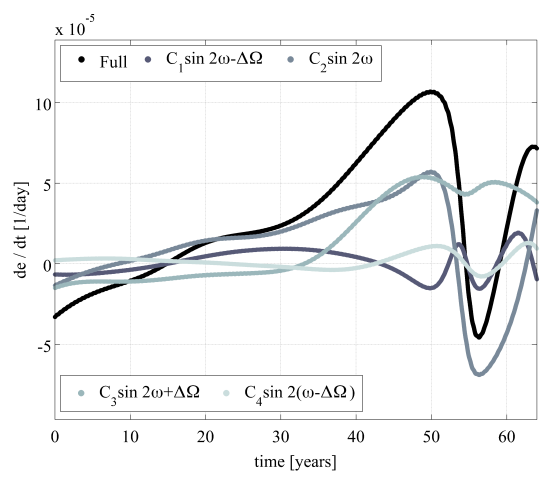

(d) Contribution of different term to eccentricity derivative

Figure 17: Analysis of eccentricity build-up for SSC 37846 with minimum time-to-reentry (Sol \#137). 


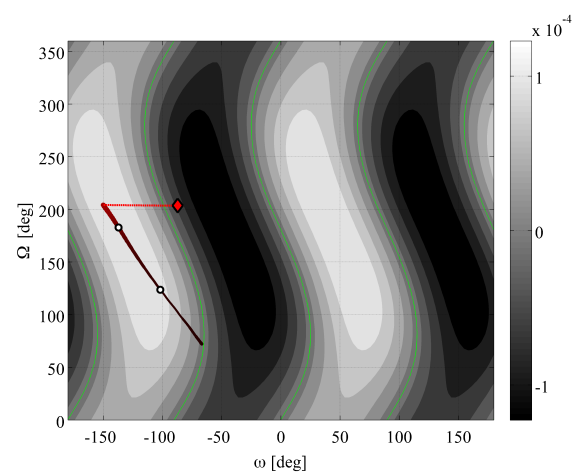

(a) Disposal map for mean orbital elements

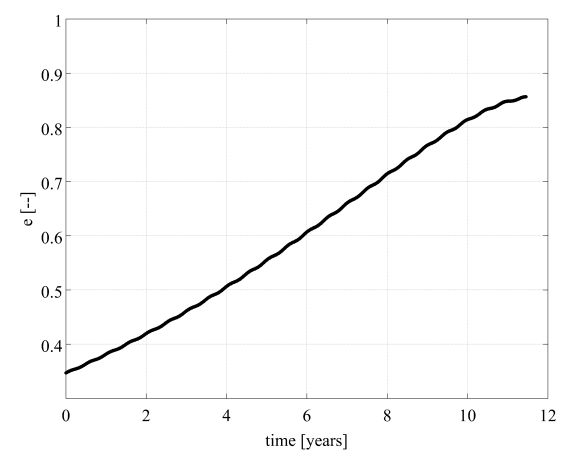

(c) Evolution of eccentricity

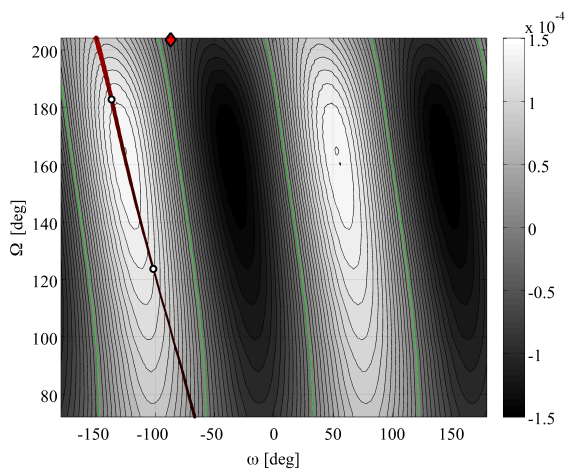

(b) Disposal map for orbital elements evolution

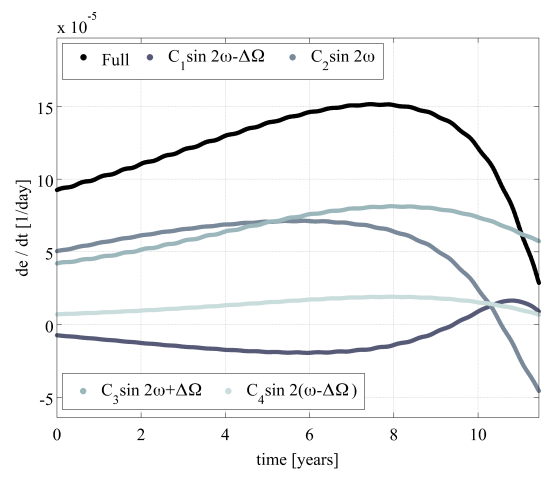

(d) Contribution of different term to eccentricity derivative

Figure 18: Analysis of eccentricity build-up for SSC 41775 with minimum time-to-reentry (Sol \#200). 


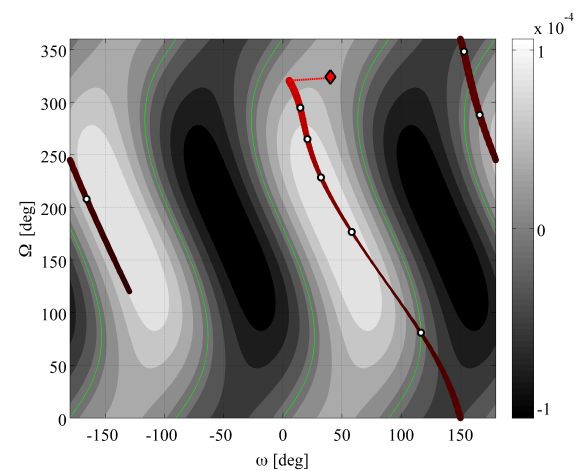

(a) Disposal map for mean orbital elements

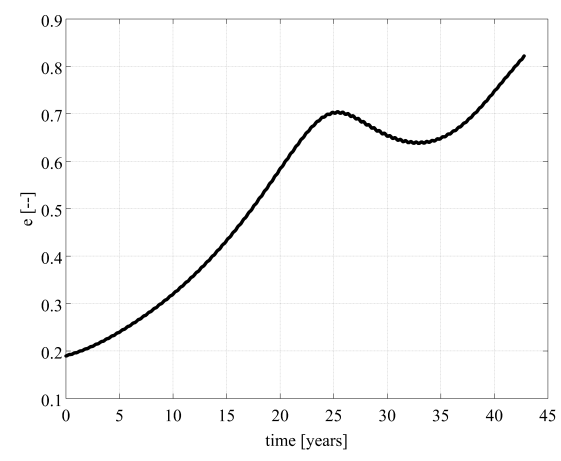

(c) Evolution of eccentricity

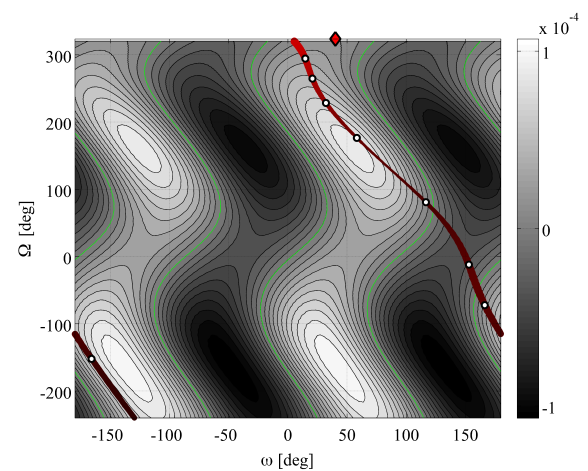

(b) Disposal map for orbital elements evolution

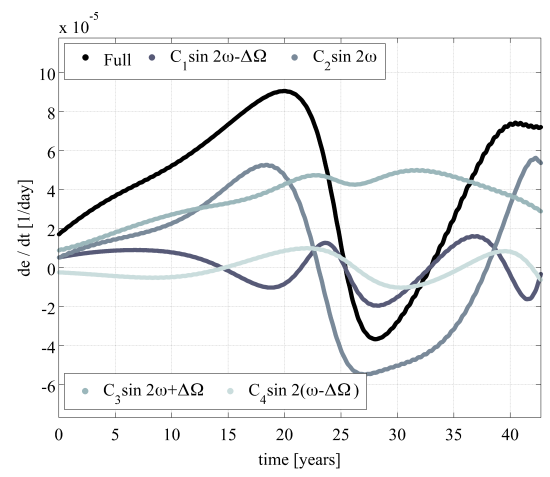

(d) Contribution of different term to eccentricity derivative

Figure 19: Analysis of eccentricity build-up for SSC 40980 with minimum time-to-reentry (Sol \#149). 


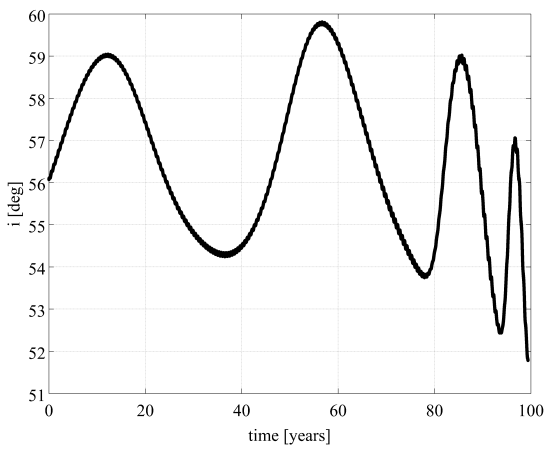

(a) Sol \#1

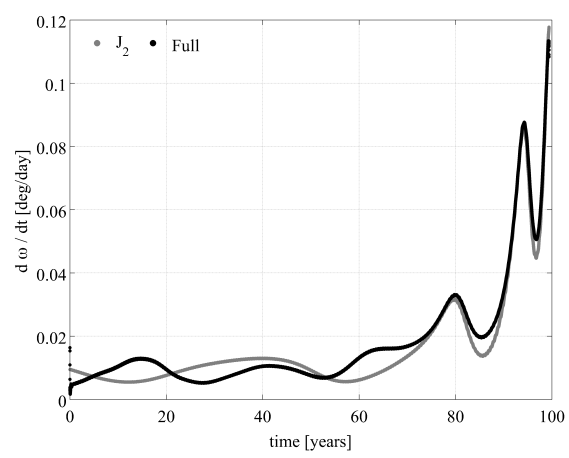

(c) Sol \#1

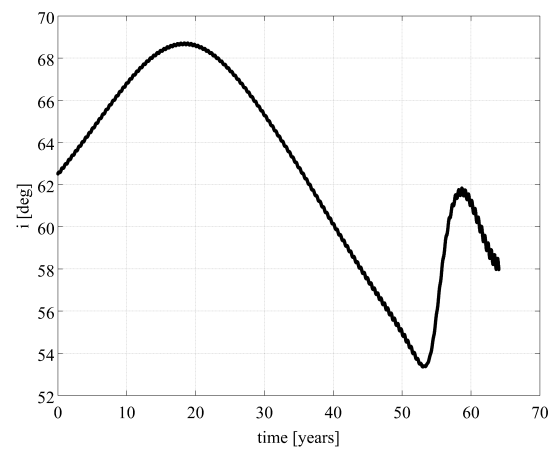

(b) Sol \#137

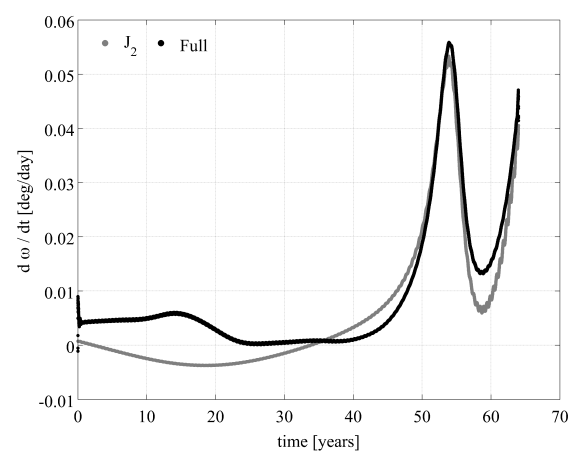

(d) Sol \#137

Figure 20: Analysis of inclination and $\dot{\omega}$ for SSC 37846 . 